\title{
Low-power Suppression of Fast-Motion Spin 3/2 Signals
}

\author{
Evgeny Nimerovsky ${ }^{1}$, Andrew $\mathrm{J} \mathrm{Ilott}^{1}$ and Alexej Jerschow ${ }^{1 *}$ \\ ${ }^{1}$ Department of Chemistry, New York University, New York, NY 10003, USA \\ *corresponding author:email: alexej.jerschow@ nyu.edu; Phone: 1-212-998-8451 \\ Department of Chemistry, New York University, 100 Washington Square East, New York, NY 10003, USA
}

\begin{abstract}
Triple Quantum Filters (TQFs) are frequently used for the selection of bi-exponentially relaxing spin 3/2 nuclei (in particular ${ }^{23} \mathrm{Na}$ ) in ordered environments, such as biological tissues. These methods provide an excellent selection of slow-motion spins, but their sensitivity is generally low, and coherence selection requirements may lead to long experiments when applied in vivo. Alternative methods, such as 2P DIM, have demonstrated that the sensitivities of the signals from bi-exponentially relaxing sodium can be significantly increased using strategies other than TQFs. A shortcoming of this method in particular is its strong dependence on $\mathrm{B}_{0}$ inhomogeneities. We describe here a method, which is sensitive to the slowmotion regime, whereas the signal from spins in the fast-motion regime is suppressed. This method is shown to be more effective than TQFs, requires minimal phase cycling for the suppression of the influence of $\mathrm{rf}$ inhomogeneity, and has less dependence on resonance offsets and $\mathrm{B}_{0}$-inhomogeneity than 2P DIM.
\end{abstract}

\section{Introduction}

Nuclear spins larger than $1 / 2$ experience a quadrupolar interaction, which can be used for characterizing the electric field gradient around them. In semi-solids, the interaction often fluctuates and leads to relaxation. In spins $3 / 2$, this process manifests itself in a bi-exponential relaxation mechanism ${ }^{1}$, wherein two $T_{1}$ and two $T_{2}$ time constants can be observed. Triple Quantum Filters (TQFs) have long been used for the separation between mono- and bi-exponential behaviors due to their ability to provide, in principle, a clean separation of signals. Despite their adequate performance in a spectroscopy setting, they suffer from drawbacks which make them less desirable for imaging, especially in vivo: their sensitivity is generally low, and longer phase cycles frequently need to be used, which add a significant overhead to the implementation. Pulsed field gradients for coherence selection are not a viable option in vivo. Here we show a simple alternative, which provides a more sensitive selection of the slow-motion relaxation regime. Our focus is in particular on rf power limited applications, which are relevant in the context of in vivo MRI and ex situ NMR experiments.

Specifically, we demonstrate the methodology on sodium spins. Sodium ions represent the most important abundant cations in the body, and perform essential physiological functions. It tissues, both fast-motion and slow-motion sodium pools are present, with the former often attributed to the extracellular environment, and the latter to the intra-cellular compartment. ${ }^{2,3}$ Certain extracellular environments have also been shown to exhibit a slow-motion regime, for example in the heart ${ }^{4}$. In cartilage, because of attraction of sodium to polyanions, sodium shows in addition a residual quadrupolar splitting. Optimal sequences for the case of selecting signals from compartments with such splittings have been described recently with a low-power pulse sequence. ${ }^{5}$ 
The possibility of differentiating between intra- and extracellular sodium is of interest, since the change in intracellular sodium concentration is an accompanying factor of many diseases and pathologies ${ }^{3}$. Often, it is assumed that the slow-motion regime is mostly prevalent in the intracellular environment, and not in the extracellular environment, which can form the basis of a selection, although this is not always the case. The general methods for the selection and measurement of the signal from the slow-motion sodium pools are the $\mathrm{TQF}^{6,7}$ and the Double Quantum Filter (DQF) sequences ${ }^{8,9}$. Other options include a weighted Inversion Recovery technique ${ }^{10,11}$. Chemical shifts between the two regimes can in some cases be observed with the help of shift agents, but these are toxic and do not represent a viable option in most situations $^{12}$. The common shortcomings of the TQF MRI method in an imaging setting are the low Signalto-Noise Ratio ${ }^{13,14}$ (SNR) and long phase cycles. Sensitivity is further reduced in rf-power-limited applications ${ }^{15}$. For this reason, it has been proposed, for example, to use TQF without a refocusing pulse ${ }^{16-18}$. In that case, the presence of $\mathrm{B}_{0}$ inhomogeneity can reduce $S N R$ further. The long phase cycles require high stabilities to avoid unwanted signals leaking through ${ }^{19}$, in particular when there is motion involved, such as in CSF. The use of pulsed field gradients ${ }^{7,20}$ is not a suitable option for MRI ${ }^{19}$.

Two new methods were published recently: three ${ }^{21}$ and two ${ }^{22}$-pulse bi-exponential-weighted ${ }^{23} \mathrm{Na}$ sequences, which allow to significantly increase SNR of the slow-motion sodium signals. The methods rely on a two-step process. The signal obtained from a $90^{\circ}$-pulse contains the contribution from all sodium fractions. In a second scan, the signal is obtained using two $90^{\circ}$-pulses $\left(2 \mathrm{P} \mathrm{DIM}^{22}\right)$ separated by a delay. The signal of fast-motion sodium can then be separated out by taking the difference between the first signal and the second signal. The second signal has to be modified by a weighting factor to adjust for a reduction of the signal due to $T_{2}$-relaxation, however. On the other hand, the SNR of this method can be about 10 times larger than the one of TQF. The major shortcoming of this method is the strong dependence on resonance offsets and hence $\mathrm{B}_{0}$ inhomogeneity. The difference and weighting procedures also introduce noise. Since $T_{2}$ and not $T_{2}^{*}$ is used for the calculation of the weighting factor in the presence of inhomogeneity, the signal from the fast-motion sodium does not vanish completely after subtraction of the two parts. For the specific case of interest where one wishes to distinguish between intra- and extracellular sodium, one should also consider that the latter has a concentration that is about 10 times larger than the former. In such a case, the undesired signal obtained with 2P DIM would be about equal or even stronger relative to the signal of intra-cellular sodium given typical inhomogeneities.

Using a similar idea, a one-pulse short- $\mathrm{T}_{2}{ }^{23} \mathrm{Na}$ imaging method was described ${ }^{23}$. In that case, the second part consisted simply of a regular one- $90^{0}$-pulse experiment with delayed acquisition (DR DIM ${ }^{22}$ ). The difference between their method and DR DIM is that the authors did not use the weighting factor and performed an optimization of the delay time. The delay time is chosen such that the decay of the fastmotion sodium signal is minimal while the decay of the intra-cellular sodium signal is maximal. Even with equal concentrations for fast- and slow-motion pools, the separation is not very good, however (Fig.5 in Reference ${ }^{23}$ ).

In the following, we describe a Fast-Motion Suppressed sequence (FMS), based on a quadrupolar jumpand-return sequence ${ }^{24}$ (Figure 1). The sequence is shown to provide adequate suppression when the characteristic delay $\tau$ is small compared to the spin-lattice relaxation time $T_{1}$, and the $T_{1}$ is not much longer than the spin-spin relaxation time $T_{2}$, which typically is the case for the fast-motion regime, and significantly distinct from the parameters of the slow-motion regime. This method only requires two-step phase cycling for the suppression of rf-inhomogeneity artifacts and has less dependence on offsets and $\mathrm{B}_{0}$-inhomogeneity with respect to 2P DIM. The experiments were also performed using a weak rf-field, to emphasize that this method could specifically be used under power-limited conditions, such as typically found for in-vivo MRI. 


\section{Theory and Simulations}

Fig. 1a shows a Jump-Return pulse block which consists of two pulses with flip angle $\beta$ and opposite phases $^{24}$, separated by a delay $\tau$. The FMS sequence (Fig. 1b) consists of a pair of the same pulses and an additional $180^{\circ}$-pulse in the middle of the delay.

(a)

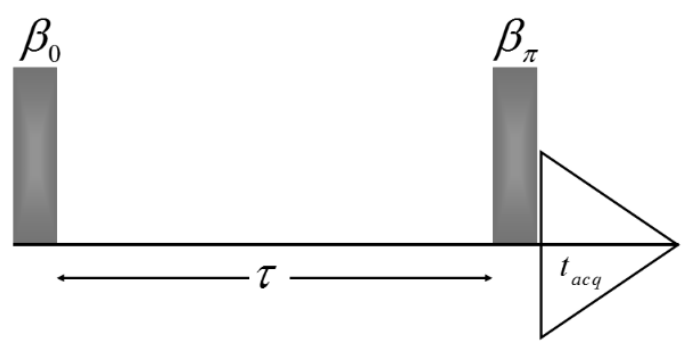

(b)

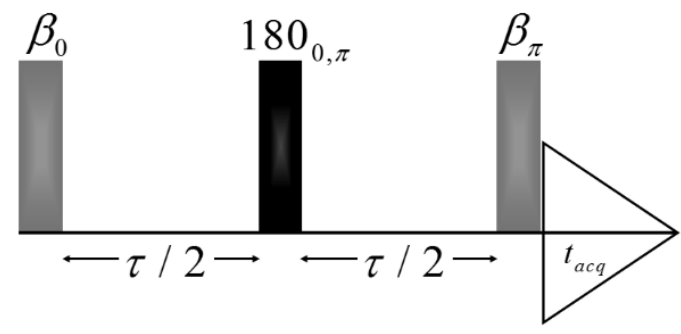

Fig. 1 (a) Jump-and-Return block. (b) FMS sequence.

Key to the selection process in this sequence is exploiting the difference between longitudinal and transverse relaxation. In the absence of relaxation, and any evolution during the delay, a jump-and-return sequence (Fig. 1a) would (ideally) produce no signal (the magnetization trajectory is depicted in Figs. 2a$\mathrm{d}$ for illustration), because the magnetization is simply flipped back by the second pulse.

In the presence of relaxation (fast-motion regime) Figs. 2e-g demonstrate how a non-zero signal could be obtained, due to a decrease in the angle between the magnetization and the $\hat{z}$-axis during the delay time (Fig. 2f). The magnetization is not flipped back by the second pulse, since $\beta^{\prime}<\beta$. The non-zero signal from fast-motion spins is obtained after the second pulse (Fig. 2g).

This non-zero signal can be minimized by the additional $180^{\circ}$-pulse in the middle of the delay as illustrated in the trajectories of Figs. 2h-k. During the first part of the delay ( $\tau / 2$, Fig. 2h) the values of $M_{z}$ and $M_{y}$ progress similarly to what is shown in Fig. $2 \mathrm{f}\left(\beta_{1}<\beta\right)$. The $180^{\circ}$-pulse inverts these components (Fig. 2i). During the second part of delay, the value of $M_{z}$ decreases (Fig. 2j) due to $T_{1}$ relaxation, which increases the angle between the magnetization and the $\hat{z}$-axis, thus compensating for the decrease in angle during the first part of the delay. The range of values of the delay can therefore be chosen such that before the last pulse $\beta_{2}$ can be approximately equal $\beta$. Therefore, after the third pulse, the final measured signal can become small again (Fig. 2k), which illustrates how fast-motion sodium signals could be suppressed.

The efficiency of this compensating mechanism obviously depends on the difference between $T_{1}$ and $T_{2}$ and the ratio between the delay time and the spin-lattice relaxation $\tau / T_{1}$. One can therefore determine a 
maximum value for the delay, $\tau_{\max }$, above which the suppression of the fast-motion signal is no longer satisfactory. This is further explored in simulations below.

(a)

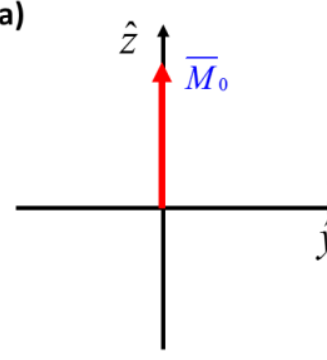

(b)

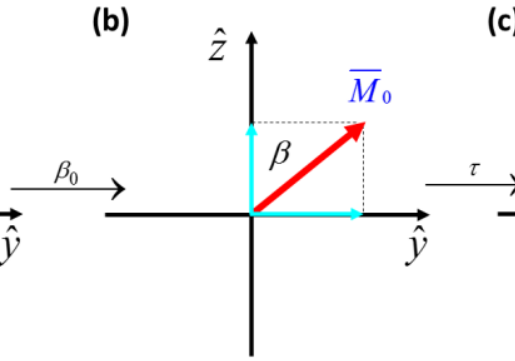

(c)

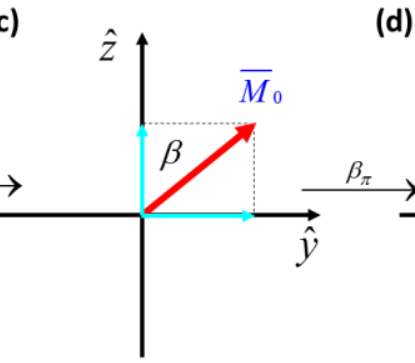

(d)

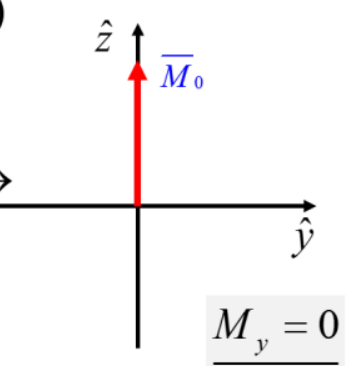

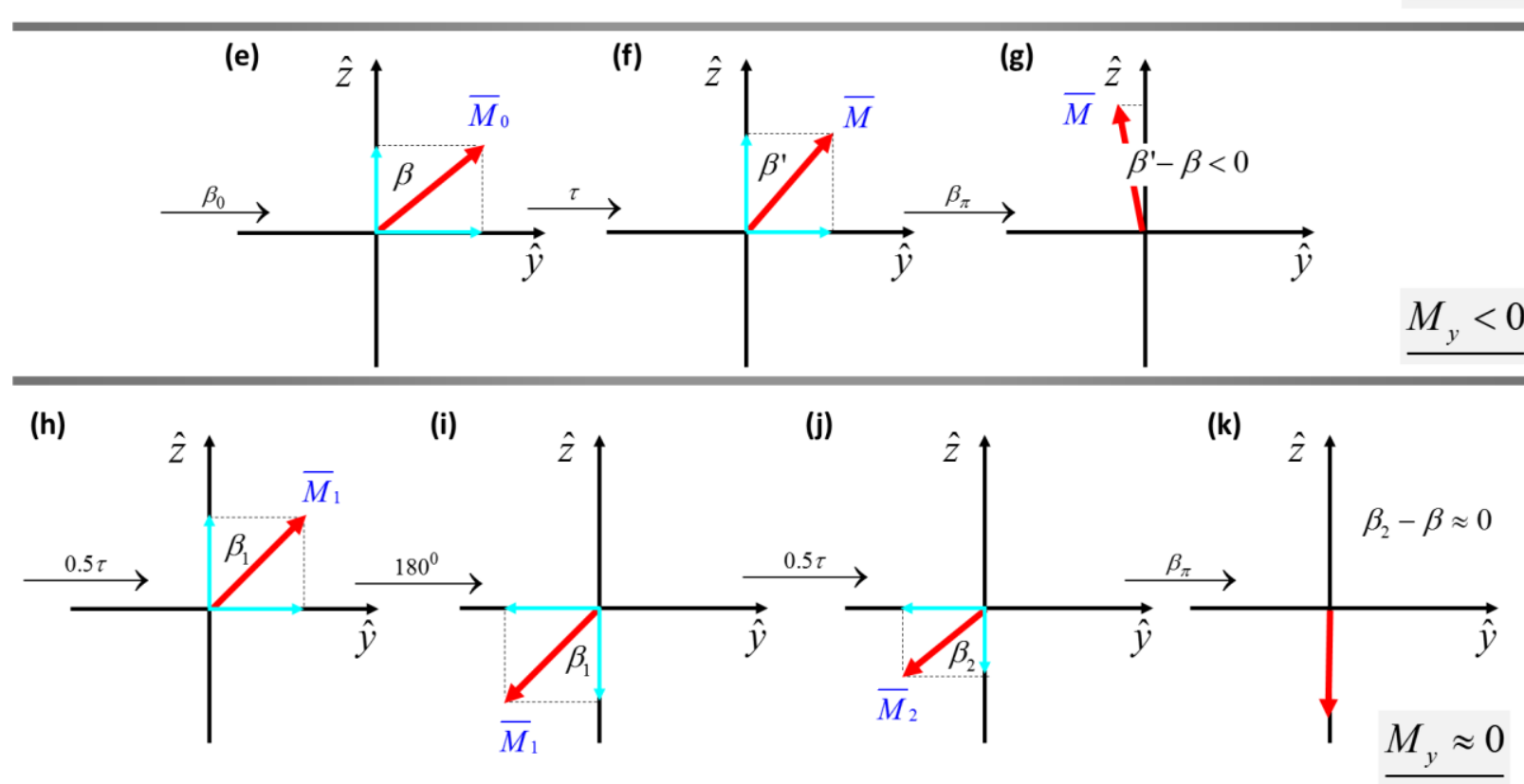

Fig. 2 Schematic description of the evolution of the magnetization in the $y-z$ plane during the Jump-and-Return block ((a)-(g)) (Fig. 1a) and the FMS sequence ((h)-(k)) (Fig. 1b). In (a)-(d), relaxation effects are neglected. The other diagrams include monoexponential relaxation with the condition $T_{1}=T_{2}$. The red arrow represents the vector magnetization. Cyan arrows show the magnetization components in the directions $\hat{y}$ and $\hat{z}$.

By the same analysis, one can show that in the case of the slow-motion regime in general and not only in the case of bi-exponentially relaxing spins, $\left(T_{2}<<T_{1}\right)$, the signal can be significant, since the transverse magnetization decays very quickly, and the angle between the magnetization and the z-axis becomes very small before the last pulse. This effect will lead to an enhanced discrimination between the two regimes.

An analysis based on spherical tensors transformations offers more detail of the selection process. In the case of bi-exponential relaxation at the conclusion of the Jump-and-Return block (Fig. 1a) one obtains the following state:

$$
\begin{aligned}
& S\left(\tau ; t_{a c q}\right)=\sin (\beta)\left\{\cos (\beta)\left[f_{11}^{(1)}(\tau)-f_{11}^{(0)}(\tau)\right]+f_{11}^{(0)}(\tau)-1\right\} f_{11}^{(1)}\left(t_{a c q}\right)- \\
& -\left\{\frac{1}{8} \sin (2 \beta)\left(11-15 \cos ^{2}(\beta)\right) f_{31}^{(1)}(\tau)-\frac{\sqrt{6}}{4} \sin (\beta)\left(5 \cos ^{2}(\beta)-1\right)(1-\cos (\beta)) f_{31}^{(0)}(\tau)\right\} f_{31}^{(1)}\left(t_{a c q}\right)
\end{aligned}
$$


where $f_{11}^{(1)}(t)=1 / 5\left[3 e^{-\left(J_{0}+J_{1}\right) t}+2 e^{-\left(J_{1}+J_{2}\right) t}\right], f_{31}^{(1)}(t)=\sqrt{6} / 5\left[e^{-\left(J_{0}+J_{1}\right) t}-e^{-\left(J_{1}+J_{2}\right) t}\right], f_{11}^{(0)}(\tau)=$ $1 / 5\left[e^{-2 J_{1} \tau}+4 e^{-2 J_{2} \tau}\right], f_{31}^{(0)}(\tau)=2 / 5\left[e^{-2 J_{1} \tau}-e^{-2 J_{2} \tau}\right]$ and $J_{0}, J_{1}, J_{2}$ are the spectral densities ${ }^{25}$. The derivation of Eq. (1) is shown in Appendix A, Eqs. (A1)-(A7).

The single quantum coherences $\left(T_{1, \pm 1}\right)$ have a maximum amplitude for $\beta=45^{0}$. For the third rank antiphase single quantum coherences $\left(T_{3, \pm 1}\right)$, the maximum amplitude of the first term, which depends on $f_{31}^{(1)}(\tau)$, is obtained for $\beta=63.5^{0}$. The second term (which depends on $f_{31}^{(0)}(\tau)$ ) equals almost zero with this flip angle. Therefore, an optimal flip angle can be chosen between $\beta \epsilon$ [45: 65] in order to provide a good signal for bi-exponentially relaxing spins.

In the fast-motion regime, i.e. when $T_{1}=T_{2}$, we have $f_{11}^{(1)}=f_{11}^{(0)}=f(\tau)=e^{-\tau / T_{1}}=e^{-\tau / T_{2}}$, and Eq. (1) equals to

$$
S\left(\tau ; t_{a c q}\right)=\sin (\beta)\{f(\tau)-1\} f\left(t_{a c q}\right) .
$$

If $\tau / T_{1} \ll 1$ the difference $f(\tau)-1 \approx 0$ and the signal can be neglected. With increasing ratio $\tau / T_{1}$, the signal will also increase and will reach the value of $-\sin (\beta)$ when $\tau / T_{1} \gtrsim 5$. In Fig. 3a we show the signal intensity (Eq. (2)) of mono-exponentially relaxing sodium with relaxation times $T_{1} \approx T_{2}=50 \mathrm{~ms}$ (the lowest approximate relaxation time of sodium in CSF in brain ${ }^{13}$ ) as a function of $\tau$ (red thin line). The intensity was normalized by the signal from a $90^{\circ}$ hard pulse excitation. The flip angles of the jump-andreturn pulses were chosen as $\beta=51^{0}$ in the experiments, where this value was found to be optimal. In Fig. $3 b$ we show the signal dependence on the flip angle $\beta$. In both cases, with growing $\tau$ or $\beta$, the intensity of the undesired signal also grows significantly. At $\tau=14 \mathrm{~ms}$, the signal reaches a value of $20 \%$ with respect to the reference signal $\left(90^{0}\right.$-excitation).
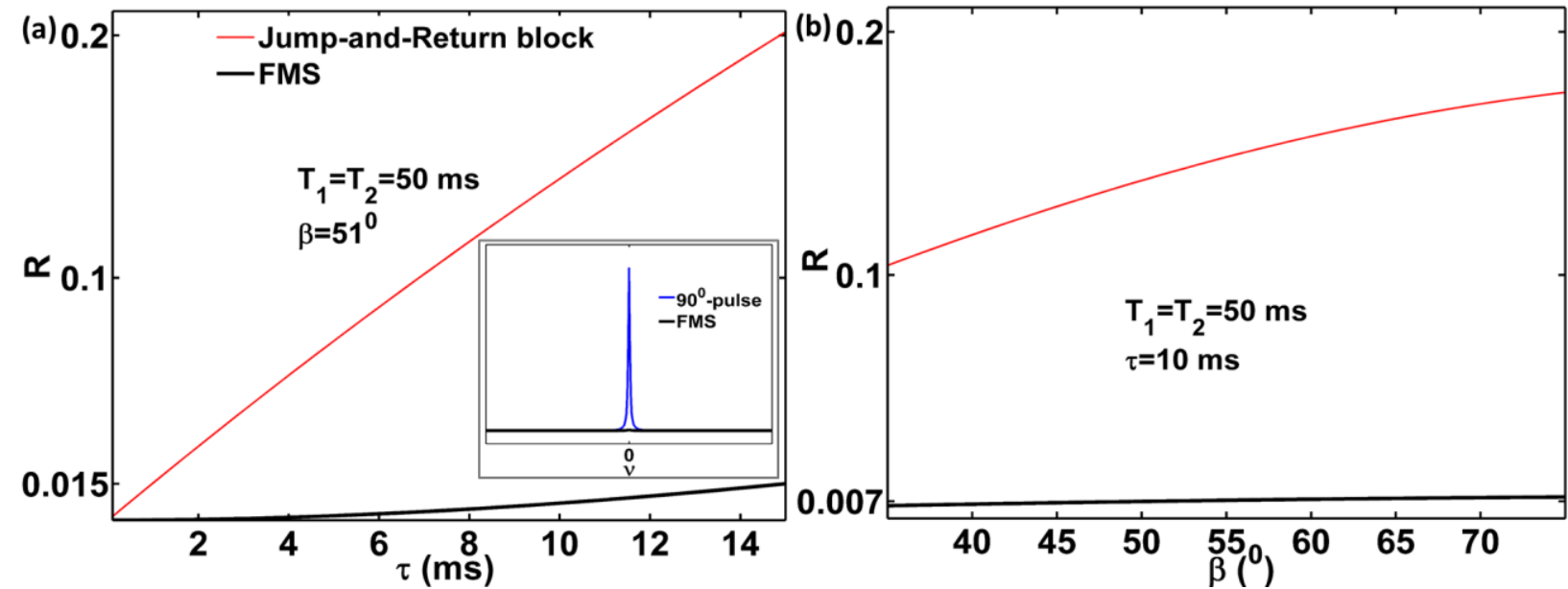

Fig. 3 Normalized signal of mono-exponentially relaxing spins as a function of delay time $\tau$ (a) and flip angle of the jump-return pulses $\beta$ (b). (a) The flip angle is constant and is set to $51^{\circ}$. (b) The delay time is constant and is chosen as $10 \mathrm{~ms}$. Red thin line Jump-and-Return block, black thick line - FMS. In both cases the relaxation times for mono-exponentially relaxing spins $\left(T_{1}, T_{2}\right)$ equal to $50 \mathrm{~ms}$. The insert in (a) shows a comparison of the spectra of mono-exponentially relaxing spins after ideal $90^{\circ}$-pulse (blue line) and FMS (black thick line, $\tau=10 \mathrm{~ms}, \beta=51^{0}$ ) excitation.

When adding a $180^{\circ}$-pulse as shown in the FMS sequence in Fig. 1b, the following signal is obtained: 


$$
\begin{aligned}
& S\left(\tau ; t_{a c q}\right)=-\sin \beta\left\{\cos (\beta)\left[f_{11}^{(1)}(\tau)-f_{11}^{(0)}(\tau)\right]+1+f_{11}^{(0)}(\tau)-2 f_{11}^{(0)}(0.5 \tau)\right\} f_{11}^{(1)}\left(t_{a c q}\right)+ \\
& +\left\{\frac{1}{8} \sin (2 \beta)\left(11-15 \cos ^{2}(\beta)\right) f_{31}^{(1)}(\tau)-\frac{\sqrt{6}}{4} \sin (\beta)\left(5 \cos ^{2}(\beta)-1\right)\left[(1-\cos (\beta)) f_{31}^{(0)}(\tau)-2 f_{31}^{(0)}(0.5 \tau)\right]\right\} \times \\
& \times f_{31}^{(1)}\left(t_{a c q}\right)
\end{aligned}
$$

The derivation is shown in Appendix A, Eqs. (A8)-(A13).

As in the previous case, the optimal flip angle can be chosen between $\beta \epsilon$ [45: 65].

In the case of mono-exponential relaxation and $T_{1}=T_{2}$, Eq. (3) becomes:

$$
S\left(\tau ; t_{a c q}\right)=-\sin \beta\{1+f(\tau)-2 f(0.5 \tau)\} f\left(t_{a c q}\right) .
$$

The signal intensity is shown in Fig. 3a (black thick line) and it is seen that the undesired signal (fastmotion spins) has much less dependence on the delay time and $\beta$. At $\tau=15 \mathrm{~ms}$ it reaches an intensity of $1.5 \%$ with respect to the ideal $90^{\circ}$-pulse excitation.

Since the concentration of extracellular sodium is about 10 times larger than the one of intracellular sodium, exquisite suppression of the signals from the undesired compartment (extracellular) is necessary. The delay time $\tau$ can be chosen as no more than $6 \mathrm{~ms}$ or $\tau / T_{1} \leq 0.12$. In this case, the signal intensity from undesired spins reaches no more than $0.26 \%$ with respect to $90^{\circ}$-excitation or $2.6 \%$ in the case of ten-fold excess of concentration. The undesired signal can be suppressed further, using a shortened recycle delay TR.

So far we considered the FMS signal from mono-exponentially relaxing spins in the fast-motion regime taking into account that $T_{1}=T_{2}$. In real systems, there is some deviation of $T_{2}$ from $T_{1}$. In this case $\left(T_{1} \neq\right.$ $T_{2}$ ) and Eq. 3 can be written as the follows:

$$
S\left(\tau ; t_{\text {acq }}\right)=-\sin \beta\left\{\cos (\beta)\left[\mathrm{e}^{-\tau / T_{2}}-\mathrm{e}^{-\tau / T_{1}}\right]+1+\mathrm{e}^{-\tau / T_{1}}-2 \mathrm{e}^{-0.5 \tau / T_{1}}\right\} \mathrm{e}^{-t_{a c q} / T_{2}} .
$$

Mutual cancellation of terms with opposite signs leads to a limited signal from undesired spins up to a certain level of difference between $T_{1}$ and $T_{2}$. This is examined further in Fig. $4 \mathrm{a}$ where the intensity of the signal is plotted as a function of $T_{2}$ for different values of $\tau$. For three different values of $\tau$ and for $T_{2}>41 \mathrm{~ms}$, the undesired signal stays relatively small $(<0.7 \%)$. Therefore, in the case of small distributions of the values of $T_{1}$, and $T_{2}$ the FMS sequence can also suppress the undesired signal well. It is hence not a requirement to have $T_{1}=T_{2}$ and the signal from the fast-motion regime can be relatively small if $T_{2} \sim T_{1}$ (the case of the fast-motion regime) and $\tau / T_{1} \leq 0.12-0.3$ as shown below. For large inhomogeneity, and if $\tau \ll T_{2}^{*}$, the undesired signal is still small. For $\tau \gtrsim T_{2}^{*}$ the undesired signal can have a significant value. This situation can be considered as a 'mock slow-motion regime'.

So far, we considered the behavior of the magnetization of the fast-motion regime with ideal pulses. To examine rf-inhomogeneity, we introduce an additional parameter $\alpha$ denoting the flip angle of the middle pulse. Considering the two-step phase cycle for this pulse, the measured signal of the fast-motion regime as a function of $t_{a c q}$ is 


$$
S\left(\tau ; t_{\text {acq }}\right)=-\sin (\beta)\left\{-\cos (\alpha) \cos (\beta)\left[\mathrm{e}^{-\tau / T_{2}}-\mathrm{e}^{-\tau / T_{1}}\right]+1-\cos (\alpha) \mathrm{e}^{-\tau / T_{1}}-(1-\cos (\alpha)) \mathrm{e}^{-0.5 \tau / T_{1}}\right\}
$$

The derivation of Eq. (6) is shown in Appendix B. The dependence of the signal as a function of $\alpha$ is shown in Fig. $4 \mathrm{~b}$ for three different values of $T_{2}$. The dependence of the undesired signal on inhomogeneity for a range of different $T_{2}$ values is reasonably weak and falls within acceptable levels. However, since the suppression of the undesired signal is never perfect, under strong inhomogeneity the undesired signal can become significant.
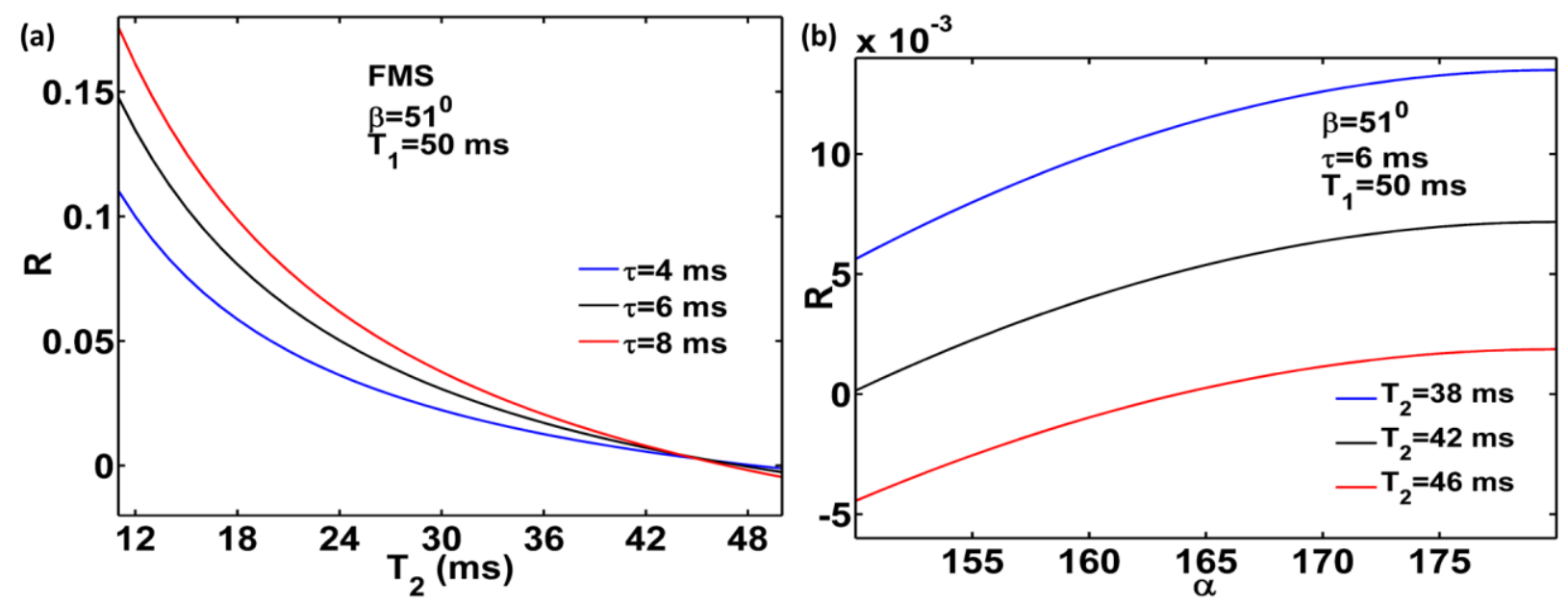

Fig. 4 Normalized signal of fast-motion spins as a function of $T_{2}$ (a), Eq. (5), and the flip angle of the middle pulse $\alpha$ (b), Eq. (6). (a) $\beta=51^{0}, T_{1}=50 \mathrm{~ms} . \tau=4 \mathrm{~ms}$ (blue line), $\tau=6 \mathrm{~ms}$ (black line) and $\tau=8 \mathrm{~ms}$ (red line). (b) $\beta=51^{0}, \tau=6 \mathrm{~ms}, T_{1}=50 \mathrm{~ms}$. $T_{2}=38 \mathrm{~ms}$ (blue line), $T_{2}=42 \mathrm{~ms}$ (black line) and $T_{2}=46 \mathrm{~ms}$ (red line).

In Fig. 5a-b, we show the signals of slow-motion sodium (Eq. (3)) as a function of flip angle and delay time. Fig. 5a shows that the first and third pulses also have a small dependence on rf-inhomogeneity for bi-exponentially relaxing spins. The measured signal is almost constant in the range of $\beta \in$ [50:65]. The deviations within this area are below 6\%. Fig. 5b shows that the optimal delay for FMS has a broad range and can be chosen between [3:6] ms, in this case. The measured signal as a function of $t_{a c q}$ for TQF $F^{6,7,25}$ and FMS (Eq. (3)) under the same relaxation conditions is shown in Fig. 5c. The relaxation parameters were taken from Reference ${ }^{18}$.
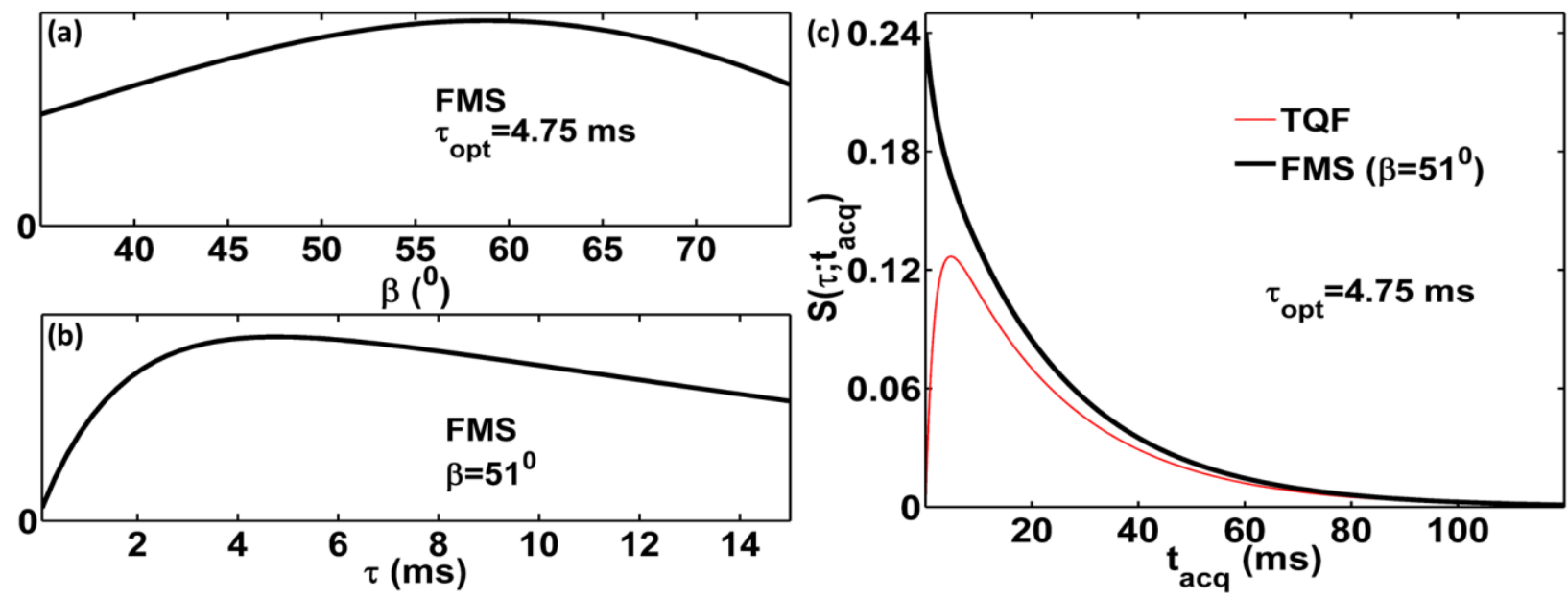
Fig. 5 (a) Dependence of the analytical FMS signal (Eq. (3), after Fourier Transform) on the delay time and the flip angle of the jump-and-return pulses (b). (a) The delay time is constant $(4.75 \mathrm{~ms})$, and (b) The flip angle is constant $\left(51^{\circ}\right)$, which is an optimal angle derived from the plot in (a). (c) Corresponding TQF (red thin line) and FMS (black thick line) signals as a function of acquisition time $t_{a c q}$. The relaxation parameters are $J_{0}=558 \mathrm{~Hz}, J_{1}=32 \mathrm{~Hz}, J_{2}=12 \mathrm{~Hz}$ in this simulation, which are the model parameters of the relaxation parameters of sodium in the brain ${ }^{18}$. The delay time in both cases is $4.75 \mathrm{~ms}$, which is an optimal time for $\mathrm{TQF}^{6}$ in this case.

RF dependence of the signals is examined in Fig. 6. With decreasing $v_{r f}$, the loss of the signal during excitation increases $^{11,15}$. For example, for $v_{r f}=0.2 \mathrm{kHz}$ (yellow thin curve), the signal reaches $50 \%$ with respect to the ideal case (black thick curve). On other hand, for $v_{r f}=0.4 \mathrm{kHz}$ (blue thin curve) or $v_{r f}=$ $0.6 \mathrm{kHz}$ (thin red curve), the loss of the signal with respect to the ideal case is not so dramatic. In these cases, the signal reaches $70 \%$ or $80 \%$, respectively.

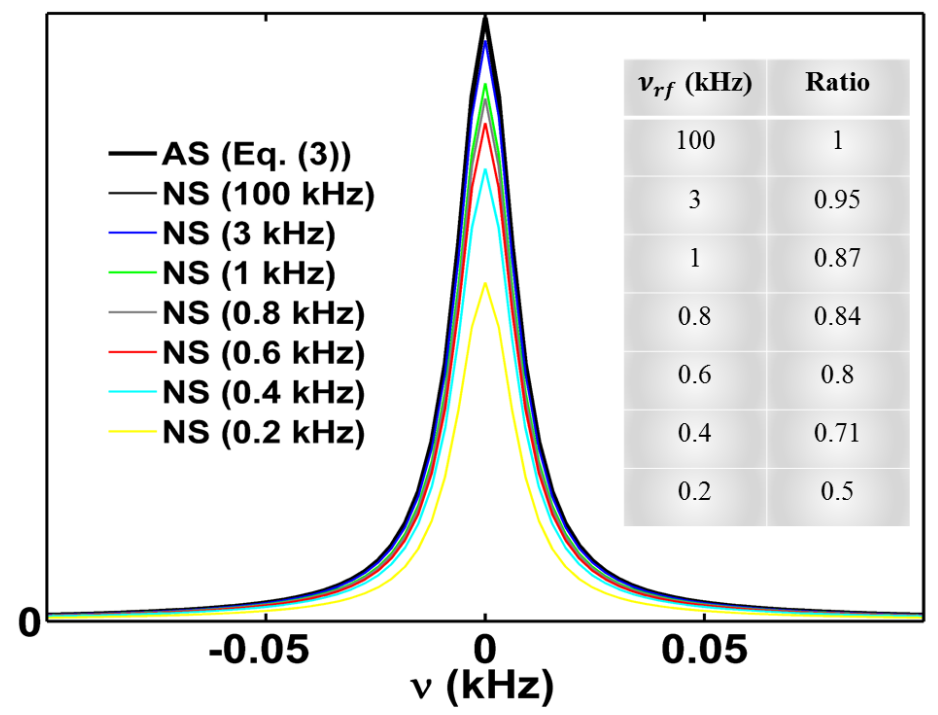

Fig. 6 FMS spectra for different values of $v_{r f}$ in the case of bi-exponentially relaxing spins. The black thick curve represents the curve from the analytical solution (AS), Eq. (3). The rest are numerical simulations (NS). The relaxation parameters are $J_{0}=$ $492 \mathrm{~Hz}, J_{1}=35 \mathrm{~Hz}, J_{2}=24 \mathrm{~Hz}$, which are the relaxation parameters of sodium in $10 \%$ of agarose ${ }^{18} \cdot \beta=51^{0}, \tau_{\text {opt }}=4.68 \mathrm{~ms}$. The normalized value was the peak with $v_{r f}=100 \mathrm{kHz}$ (black thin spectrum).

In this section we provided the qualitative and quantitative descriptions of the magnetization behavior for the fast-motion and slow-motion regimes during the FMS sequence. Based on this analysis, one can define a condition for the fast-motion regime, under which the signal from undesired spins is negligibly small whereas the signal from desired spins is significant: $\tau / T_{1} \leq 0.3\left(T_{1}, T_{2}\right.$ - relaxation times of undesired spins) in the case of equal concentrations of sodium in the fast- and slow-motion regimes, or $\tau / T_{1} \leq 0.12$ when the concentration of the former is significantly larger (ten-fold) with respect to the latter. The residual fast-motion signal theoretically is no more than $1.5 \%$ and $2.6 \%$, respectively, in the two scenarios, taking into account the difference of concentrations. This condition defines the maximum value of the delay time $\left(\tau_{\max }=\varepsilon T_{1}, \varepsilon=0.12\right.$ or 0.3 ) below which the undesired signal has a negligibly small value and is further examined in experiments. For example, in the case of mono- and biexponentially relaxing spins in brain tissues, the maximum delay $\tau$ is $6 \mathrm{~ms}$ to keep the suppression behavior acceptable. Simulations show that the selected signal from FMS is larger than the one of TQF by a factor 1.92 in the case of hard pulses and 2.54 in the case of weak pulses ( $\left.v_{r f}=300 \mathrm{~Hz}\right)$. 


\section{Experimental methods}

\section{Calculations and Simulations:}

All calculations were performed using MATLAB software. The numerical calculations were performed using a set of tensor differential equations as described in Reference ${ }^{25}$.

\section{Materials:}

3 samples of agarose $(3 \%, 5 \%$ and $10 \%)$ were prepared using standard PBS solution $(0.137 \mathrm{M}$ of $\mathrm{NaCl})$ and were packed into Quartz tubes (5 $\mathrm{mm}$ ) for NMR experiments.

For MRI experiments, a $3 \mathrm{~mm}$ glass tube with $0.5 \mathrm{M}$ solution of $\mathrm{NaCl}$ and was placed into a $5 \mathrm{~mm}$ glass tube with $5 \%$ of agarose $(0.05 \mathrm{M}$ of $\mathrm{NaCl})$.

NMR experiments:

$90^{\circ}$, FMS, TQF and 2P DIM experiments were performed using a broad-band direct observe probe tuned to sodium on a $500 \mathrm{MHz}$ Bruker Avance spectrometer. The nutation frequencies for ${ }^{23} \mathrm{Na} \mathrm{NMR}$ were calibrated using a $0.4 \mathrm{M}$ solution of $\mathrm{NaCl}$. The measured $T_{2}$-relaxation time of $\mathrm{NaCl}$ solution was 57.6 ms. Table 1 lists the experimental parameters used.

Table 1. Experimental parameters used in the $90^{\circ}$, TQF, 2P DIM and FMS experiments.

\begin{tabular}{|c|c|c|c|c|c|}
\hline Materials & Method & $\mathbf{N S}^{\mathbf{1}}$ & $\mathbf{S W}^{\mathbf{2}}(\mathbf{k H z})$ & $\mathbf{A Q}^{\mathbf{3}}(\mathbf{s})$ & $\mathbf{D 1}^{\mathbf{4}}(\mathbf{s})$ \\
\hline \multirow{2}{*}{$\mathrm{NaCl}$} & $90^{0} / \mathrm{FMS}$ & $8 / 80$ & 25 & 0.11 & 0.08 \\
\cline { 2 - 6 } & FMS/2P DIM & 16 & 25 & 0.15 & 0.5 \\
\hline \multirow{2}{*}{ agarose } & 2P DIM & 12 & 25 & 0.15 & 0.5 \\
\cline { 2 - 6 } & FMS/TQF & 24 & 25 & 0.15 & 0.5 \\
\hline
\end{tabular}

${ }^{1}$ Number of Scans; ${ }^{2}$ Spectral Width $;{ }^{3}$ Acquisition time; ${ }^{4}$ Recycle delay.

\section{MRI experiments:}

UTE (Ultra Short Echo time) and FMS experiments were performed using a broad-band direct observe probe tuned to sodium on a Bruker Avance, $500 \mathrm{MHz}$ spectrometer. Axial 2D images were acquired, without slice selection, for these experiments by applying radial readout gradients during the acquisition time $^{26}$. The images were reconstructed from 160 radial $k$-space spokes using a Kaiser-Bessel function with $w=16$ for the gridding. Table 2 lists the experimental parameters used.

Table 2. Experimental parameters used in the UTE and FMS experiments.

\begin{tabular}{|c|c|c|c|c|c|c|c|c|}
\hline Method & NS & D1(s) & TD2 $^{\mathbf{1}}$ & TD1 $^{\mathbf{1}}$ & SW2 $^{\mathbf{2}} \mathbf{( k H z )}$ & SW1 $^{\mathbf{2}} \mathbf{( k H z )}$ & $\mathbf{A Q 2}^{\mathbf{3}}(\mathbf{s})$ & AQ1 $^{\mathbf{3}}(\mathbf{s})$ \\
\hline UTE & 400 & 0.08 & 1024 & 160 & 100 & 5.162625 & 0.005175 & 0.015496 \\
\hline FMS & 1020 & 0.08 & 1024 & 160 & 100 & 5.162625 & 0.005175 & 0.015496 \\
\hline
\end{tabular}

${ }^{1}$ Number of data points in direct (2) and indirect (1) dimensions; ${ }^{2}$ Spectral Widths of direct (2) and indirect (1) dimensions; ${ }^{3}$ Acquisition times of direct (2) and indirect (1) dimensions.

\section{Results and Discussion}

Figs. 7 and 8 show experimentally that the signal from undesired spins (fast-motion regime) is negligibly small under the condition laid out in the 'Theory' section. Fig. 7 shows ${ }^{23} \mathrm{Na}$ spectra of a $\mathrm{NaCl}$ solution $(0.4 \mathrm{M})$ with different rf-fields, which demonstrate that FMS (blue thick line) minimally excites fast- 
motion sodium when the condition $\tau / T_{1} \leq 0.12$ is fulfilled. The black thin lines represent the ${ }^{23} \mathrm{Na}$ spectra after a regular $90^{\circ}$-pulse excitation. The number of scans for the FMS experiments was 10 times larger with respect to $90^{\circ}$-pulse excitation in order to show the remnant of the fast-motion sodium peak.

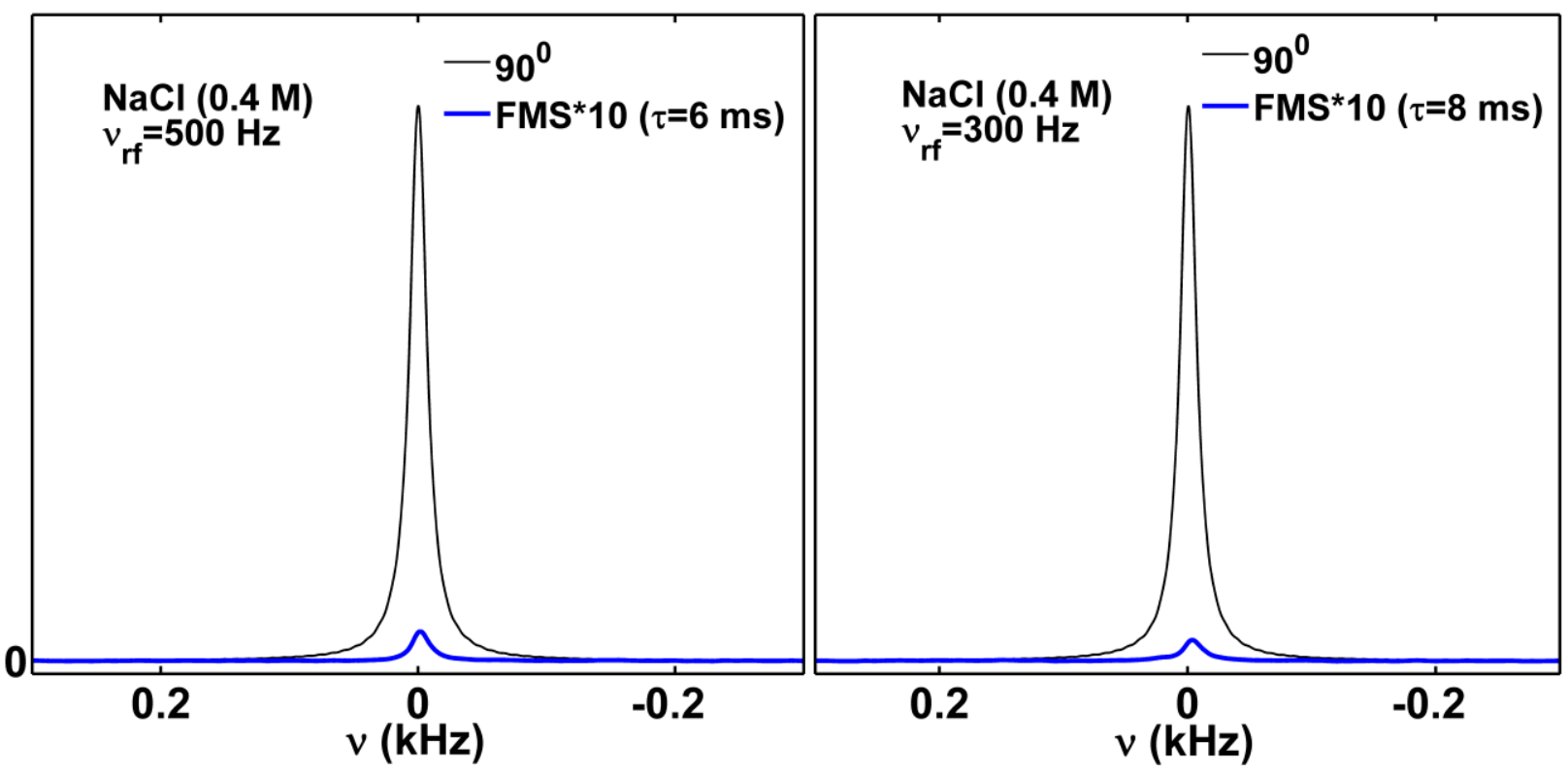

Fig. $7 .{ }^{23} \mathrm{Na}$ spectrum of $\mathrm{NaCl}(0.4 \mathrm{M})$ solution. Black line $90^{0}$ - pulse spectrum ( 8 scans $)$. Blue line FMS $\left(\beta=51^{0}, 80\right.$ scans $)$. (a) $v_{r f}=500 \mathrm{~Hz}$ The ratio between blue and black peaks is $5.3 \%$; (b) $v_{r f}=300 \mathrm{~Hz}$. The ratio between the peaks is $3.7 \%$.

In Fig. 8 we show the dependence of the normalized signal

$$
R=\text { Intensity }(F M S) / \text { Intensity }\left(90^{\circ}-\text { pulse }\right)
$$

on the delay time $\tau$, as observed from a $0.4 \mathrm{M} \mathrm{NaCl}$ solution (Fig. 8a) and the flip angle $\beta$ (Fig. 8b). The normalization factor is the signal of ${ }^{23} \mathrm{Na}$ after a $90^{\circ}$-pulse excitation. The number of scans for FMS experiments was 10 times larger with respect to the reference experiment ( $90^{\circ}$-pulse excitation).

These figures show a behavior similar to the one seen in the simulations. The dependence of the experimentally observed signal on the flip angle $\beta$ (Fig. 8b) is given by a monotonically increasing function as in Fig. 3b. When $\tau$ is in the range from $0-6 \mathrm{~ms}$ (Fig. 8a), the signals from undesired spins have negligibly small values with respect to $90^{\circ}$-pulse excitation. Since the measured $\mathrm{T}_{1}$ relaxation time of $\mathrm{NaCl}$ is $57.6 \mathrm{~ms}$, the experiments coincide with the simulations about the maximum value of $\tau$ in the regime where $\tau / T_{1} \leq 0.12$. The behavior of the signals for $v_{r f}=500 \mathrm{~Hz}$ (black line with circles) and $v_{r f}=300 \mathrm{~Hz}$ (red line with stars) in Fig.8 is similar. The small discrepancies between Fig. 8a and Fig. 3a are likely due to rf-inhomogeneity. 

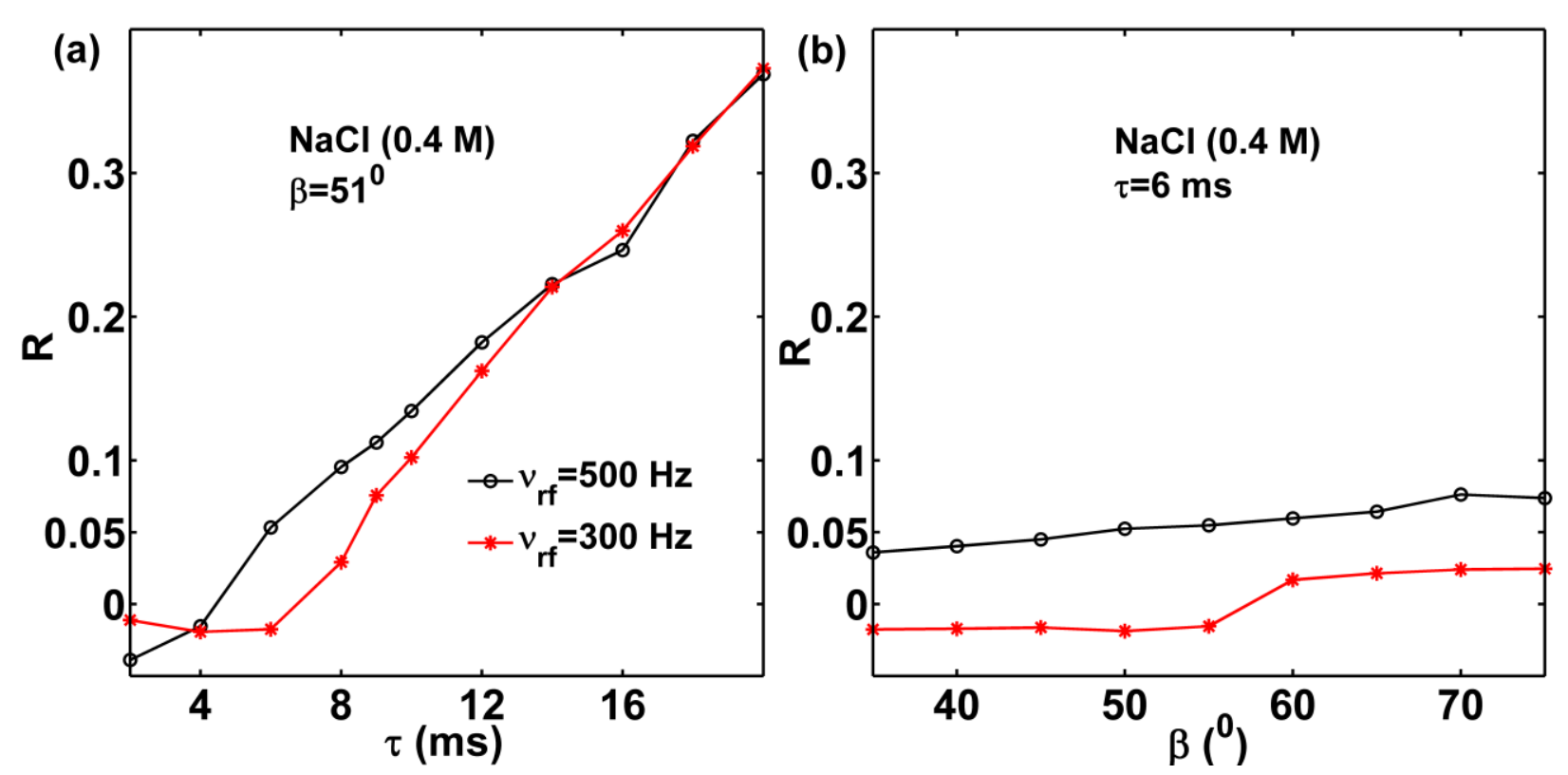

Fig. 8 Normalized FMS signal of $\mathrm{NaCl}$ solution (R) as a function of delay time ((a) flip angle is constant and equals to $\left.51^{\circ}\right)$ and flip angle ((b) delay time is constant and equals to $6 \mathrm{~ms}$ ) with $v_{r f}=300 \mathrm{~Hz}$ (red line with stars) and $v_{r f}=500 \mathrm{~Hz}$ (black line with circles). The number of scans in the FMS experiments was 80 and for the reference experiment $\left(90^{0}\right.$-pulse excitation with the same rf-field) it was 8 .

We next examine the behavior of FMS in the presence of $\mathrm{B}_{0}$-inhomogeniety. Fig. 9 shows the dependence of the normalized signals of $\mathrm{Na}$ in $\mathrm{NaCl}$ solution $(0.4 \mathrm{M})$ as a function of frequency offset for $2 \mathrm{P} \mathrm{DIM}$ (blue line with diamonds) and FMS (red line with stars), normalized over the same total number of scans.

Fig. 9 shows that 2P DIM has a very strong dependence on resonance offset. Within $\pm 40 \mathrm{~Hz}$ offset the undesired signal amounts to $40 \%$ of the reference signal. FMS has a very small dependence on resonance offset by comparison, and within $\pm 40 \mathrm{~Hz}$ the undesired signal remains below $5 \%$. As in other methods, the dependence of the FMS signal on resonance offset is expected to be symmetric around resonance. The small asymmetry visible here is likely due to pulse non-ideality. 


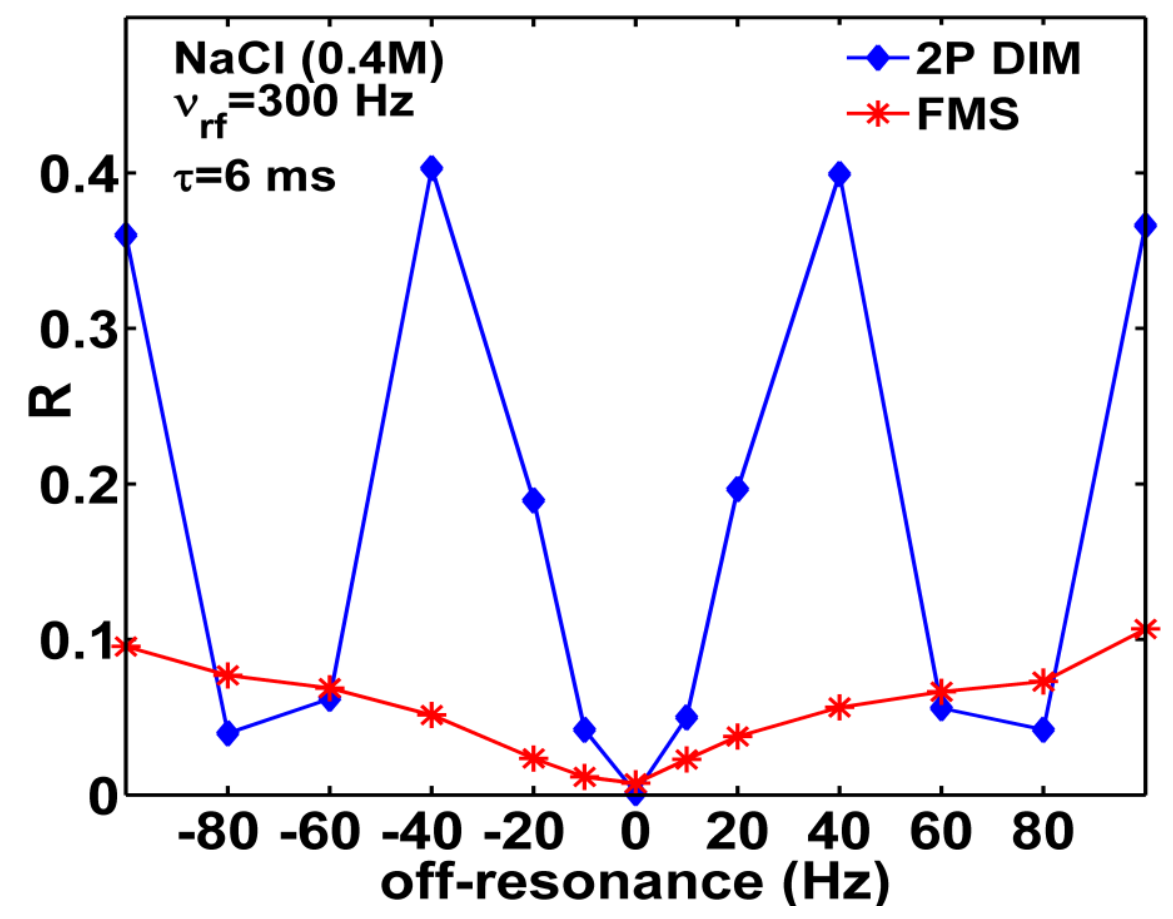

Fig. 9 The dependence of the normalized 2P DIM signal (blue line with diamonds) and FMS (red line with stars) of $\mathrm{NaCl}$ solution on resonance offset at the same experimental conditions: $v_{r f}=300 \mathrm{~Hz}, \tau=6 \mathrm{~ms}$. The flip angle for FMS was $51^{0}$.

We now turn to testing the efficiency of the method with respect to the excitation of the desired signals (slow motion regime). Fig. 10 shows ${ }^{23} \mathrm{Na}$ spectra of $3 \%, 5 \%$, and $10 \%$ agarose samples, which were obtained with 3 different methods, TQF (black line), 2P DIM (red line) and FMS (blue line). Each of the two parts of 2P DIM was performed with half the number of scans to compare the signals with the same total number of added signals (same total acquisition time). In all three cases, the FMS signals were significantly larger than in the case of TQF. With respect to 2P DIM, the FMS signals were 1.4-1.5 times smaller. As detailed further below, the advantage of the FMS with respect to 2P DIM is the much smaller dependence on the resonance offset and thus $\mathrm{B}_{0}$ inhomogeneity, as well as the independence from a weighting factor used in 2P DIM.
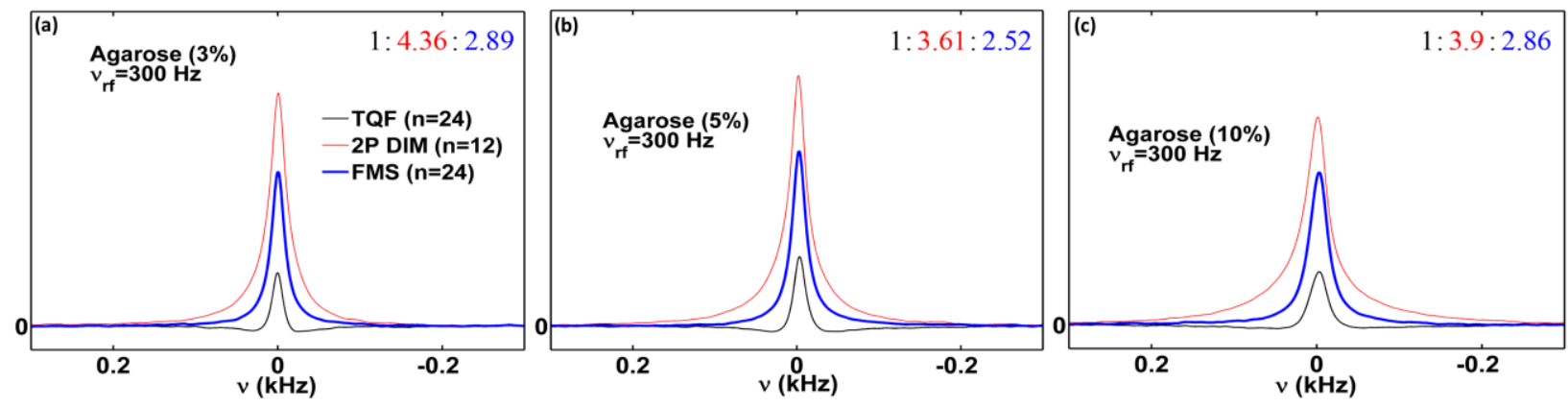

Fig. 10 TQF (black line), 2P DIM (red line) and FMS (blue line) spectra of samples with 3 different concentrations of agarose ((a)-3\%; (b)-5\%; (c)-10\%) with $v_{r f}=300 \mathrm{~Hz}$. The experimental conditions for TQF were (a) $\tau=16 \mathrm{~ms} . \mathrm{n}=24$, (b) $\tau=8 \mathrm{~ms}$, $\mathrm{n}=24$, (c) $\tau=6 \mathrm{~ms}, \mathrm{n}=24$; for $2 \mathrm{P}$ DIM (a) $\tau=10 \mathrm{~ms} . \mathrm{n}=12$, (b) $\tau=8 \mathrm{~ms}, \mathrm{n}=12$, (c) $\tau=6 \mathrm{~ms}, \mathrm{n}=12$; for FMS (a) $\tau=10 \mathrm{~ms}$. $\beta=51, n=24$, (b) $\tau=8 \mathrm{~ms}, \beta=51, \mathrm{n}=24$, (c) $\tau=6 \mathrm{~ms}, \beta=51, \mathrm{n}=24$. The comparison is based on the same total number of scans (or experiment time), because 12 scans are repeated twice for the difference experiment in $2 \mathrm{P}$ DIM.

Fig. 11 shows the dependence of the normalized signal (R, Eq. (7)) obtained from agarose solutions on $\tau$ and $\beta$ at different rf powers. The normalization factor was derived from the signal following a $90^{\circ}$-pulse 
excitation. As with other methods, the efficiency of FMS depends on pulse power. It can be seen clearly for $5 \%$ and $10 \%$ of agarose (Fig. 11c and e), whereas for $3 \%$ of agarose the difference between the signals with $v_{r f}=300 \mathrm{~Hz}$ and $200 \mathrm{~Hz}$ is small. The stronger the relaxation, the more signal is lost during the pulses ${ }^{15}$. For all three concentrations, the dependence of the signal on $\beta$ is similar to the ones predicted by the theoretical description of the sequence (Fig. 5a, b). In these cases, the signal is almost constant within the flip angle range $\beta \in\left[45^{0}: 55^{0}\right]$.

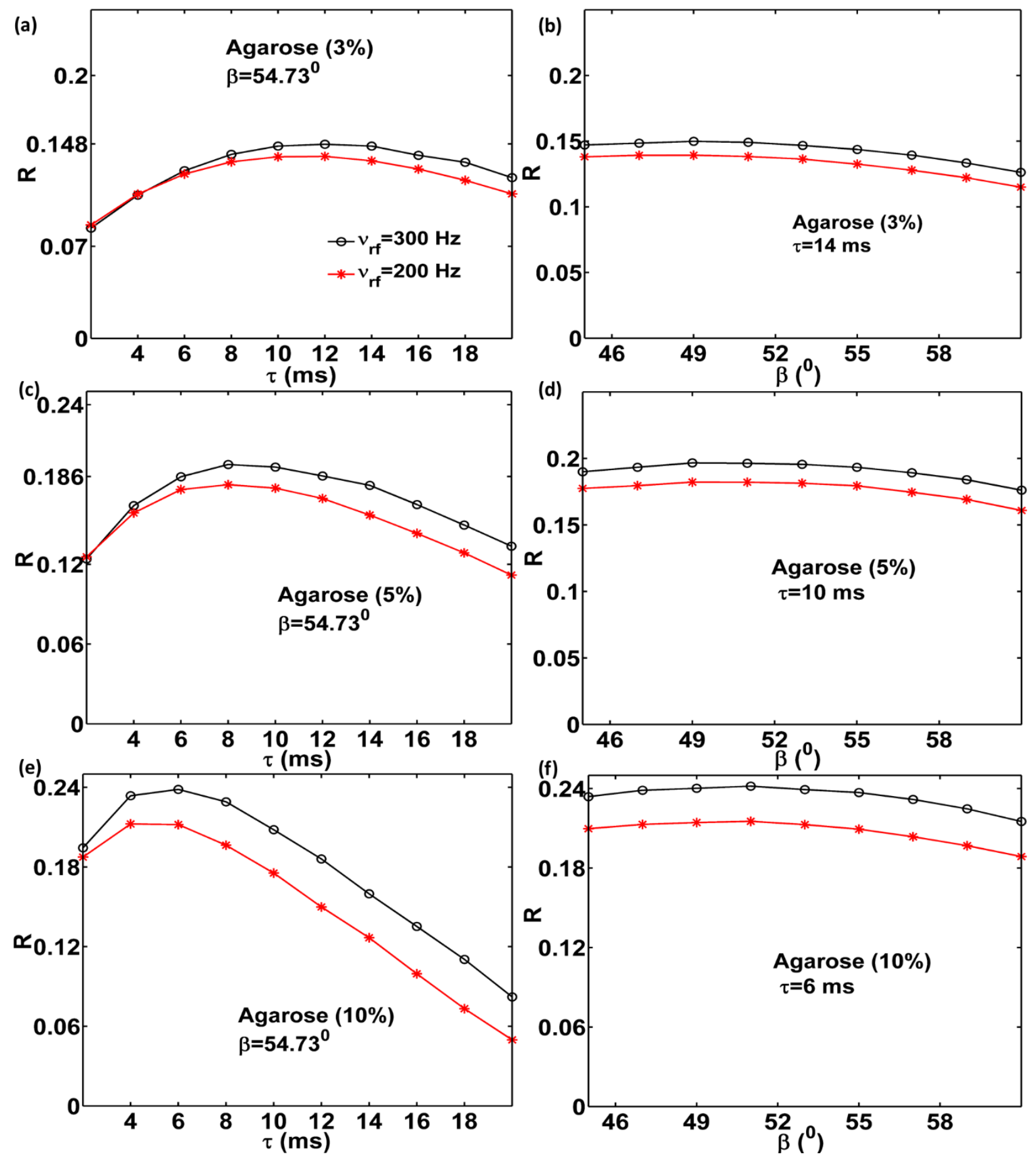


Fig. 11 Normalized FMS signal at 3 different concentrations of agarose ((a), (b)-3\%; (c), (d)-5\%; (e), (f)-10\%) as a function of delay time ((a), (c), (e) the flip angle is constant and equals to $54.7^{\circ}$ ) and flip angle ((b) $\tau=14 \mathrm{~ms}$, (d) $\tau=8 \mathrm{~ms}$, (f) $\tau=6 \mathrm{~ms}$ ). $v_{r f}=300 \mathrm{~Hz}-$ red line with stars; $v_{r f}=200 \mathrm{~Hz}$ - black line with circles.

In the previous section we showed the numerical dependence of the signal from slow-notion spins (biexponentially relaxing spins, Fig. 6a) on $v_{r f}$. Fig. 12 shows experimental ${ }^{23} \mathrm{Na}$ spectra of $10 \%$ of agarose at five different values of $v_{r f}$. The signals for powers $v_{r f}=900$ and $700 \mathrm{~Hz}$ are very similar to each other ( $85 \%$ and $84 \%$ with respect to the hard pulse signal). At $v_{r f}=500 \mathrm{~Hz}$ (blue line) the signal is at $81 \%$ with respect to the hard pulse signal. At $v_{r f}=300 \mathrm{~Hz}$ and $v_{r f}=200 \mathrm{~Hz}$ the signal has an intensity of $74 \%$ and $63 \%$, respectively. This dependence follows the same trend as seen in the simulations).

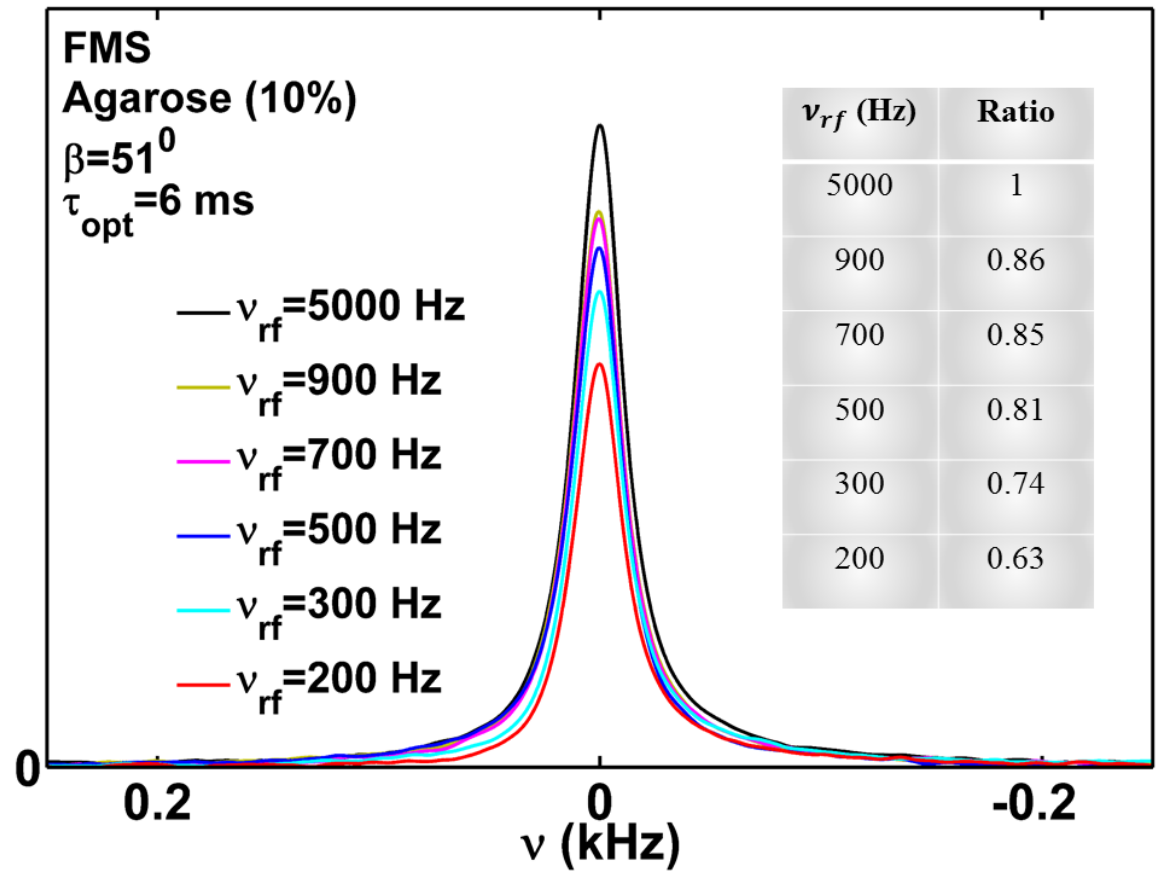

Fig. 12 FMS spectra of $10 \%$ agarose solution at different $\mathrm{rf}$ power, $v_{r f} . \tau=6 \mathrm{~ms}, \beta=51^{0}$. The peak with $v_{r f}=5000 \mathrm{~Hz}$ serves as a reference with the normalized value of 1 (black line).

Finally, we demonstrate the ability of FMS to select slow-motion sodium signals in a sample containing both environments in an image. In addition, the fast-motion compartment has a tenfold larger concentration to illustrate a case that may be prevalent in tissues. Fig. 13 shows the results from UTE MRI (a) and FMS MRI experiments (b, c), which were performed using a cylindrical sample with $0.5 \mathrm{M}$ of $\mathrm{NaCl}$ solution in the center (green circle) and $5 \%$ of agarose $(0.05 \mathrm{M}$ of $\mathrm{NaCl}$ ) surrounding it (red circle). Despite the big difference between the concentrations of sodium in the center and the surrounding volume (10:1, respectively), FMS suppresses the signal from undesired spins reasonably well at powers $v_{r f}=300 \mathrm{~Hz}$ (b) and $v_{r f}=500 \mathrm{~Hz}$ (c). These images illustrate that under conditions that would be present in vivo, such a sequence could perform quite well. 

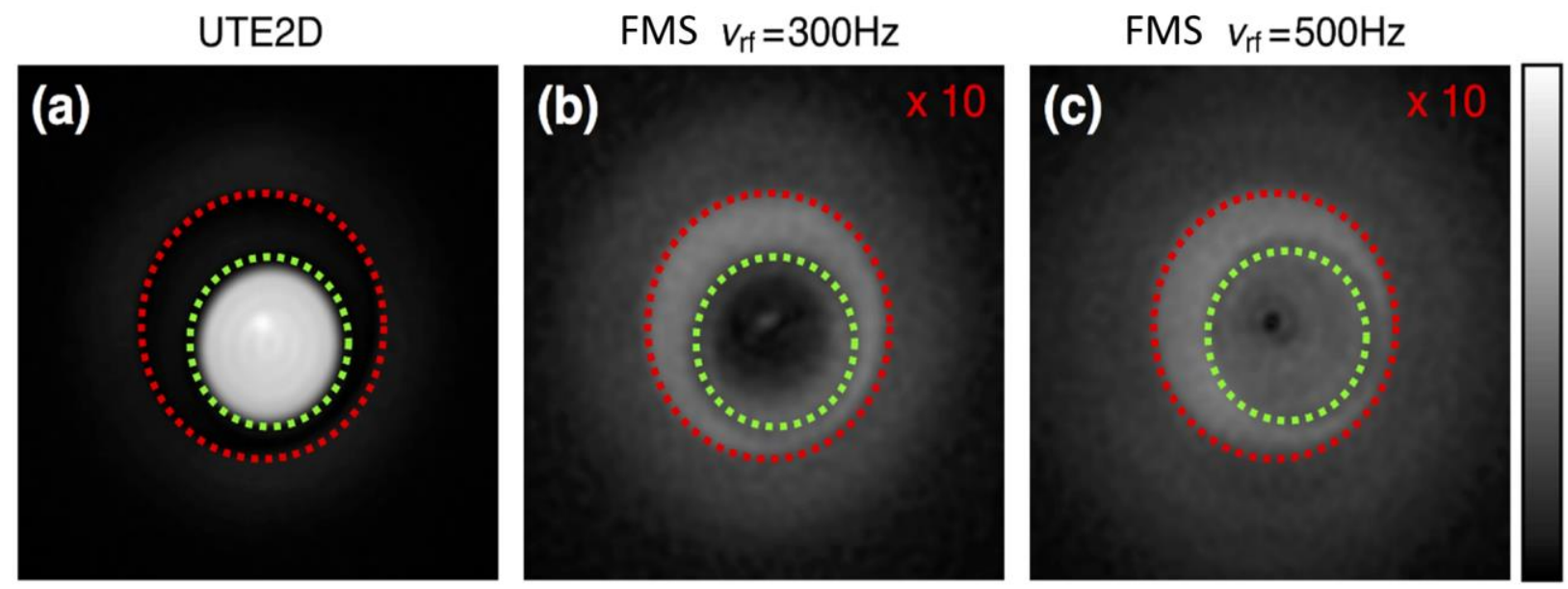

Fig. 13 UTE (a) and FMS (b, c) MRI experiments of a cylindrical sample with $\mathrm{NaCl}$ solution (0.5 M) in the center (green circle) and $5 \%$ agarose $\left(0.05 \mathrm{M}\right.$ of $\mathrm{NaCl}$ solution) around it (red circle). (a) $v_{r f}=500 \mathrm{~Hz}$; (b) $v_{r f}=300 \mathrm{~Hz}, \beta=51^{0}, \tau=6 \mathrm{~ms}$; (c) $v_{r f}=500 \mathrm{~Hz}, \beta=51^{0}, \tau=5 \mathrm{~ms}$. The image intensities in (b) and (c) have been increased by actor of 10 for better analysis.

In summary, the FMS experiments showed excellent suppression of fast-motion sodium while allowing one to observe slow-motion sodium. It was shown that FMS depended much less on $\mathrm{B}_{0}$ inhomogeneity than 2P DIM. FMS signals were shown to be about 2.5-3 times larger than TQF signals. Limitations of FMS are found with moderate to severe inhomogeneities, especially at weak power, but for those conditions currently no good alternatives exist.

\section{Conclusion}

Multiple Quantum Filters are powerful methods for the investigation of slow-motion sodium or biexponentially relaxing sodium in tissues. Their shortcomings are, however, their small signal-to-noise ratios and an overhead in experiment time due to phase cycles, especially in their MRI applications. The recently-developed technique 2P DIM, allowed one to increase SNR significantly. The design of this sequence, however, was not made with $\mathrm{B}_{0}$-inhomogeneity in mind. In this article, we showed a new method for the selective excitation of slow-motion sodium spins. The selection of the slow-motion regime with respect to the fast-motion regime performs well if $\tau / T_{1} \leq 0.3\left(T_{1}, T_{2}\right.$ - relaxation times of fastmotion spins) in the case of equal concentrations of fast- and slow-motion spins, or $\tau / T_{1} \leq 0.12$ when there is a ten-fold excess of concentration of fast-motion spins. Experiments with $\mathrm{NaCl}$ solution in agarose samples of different concentrations showed that the method can be about 2.5-3 times more efficient than TQF and only requires a two-step phase-cycle for the suppression of rf- and $\mathrm{B}_{0^{-}}$ inhomogeneities (without these effects, the sequence does not require phase cycling). Experiments and simulations showed that the optimal flip angle for jump-and-return pulses could be chosen between $\left[45^{0}: 55^{\circ}\right]$ and the optimal delay time had a broad range of values and was about the same as in the case of TQF. MRI experiments with a phantom sample showed that the sequence could suppress the signal from fast-motion spins even if their concentration was 10 times larger with respect to slow-motion spins. Although this method is less efficient than 2P DIM, it has a much smaller dependence on resonance offsets, $\mathrm{B}_{0}$-inhomogeneity, and rf-inhomogeneity. It also does not require the choice of a weighting factor as is used for 2P DIM, which has a significant influence on the final difference spectrum. Most importantly, the method can be used with a weak rf-field (with rf inhomogeneities up to $\sim 15 \%$ ). In this form, the methods could thus become useful for sodium MRI in-vivo, or in other power-limited situations.

\section{Acknowledgments}


The Bruker Avance - 500 NMR Spectrometer was acquired through the support of the National Science Foundation under Award Number CHE 01162222. We acknowledge funding from the U.S. National Science Foundation, award No. CHE 1412064 for methodology development, and NIH R01AR060238 and NIH R01EB016045 for imaging-related work.

\section{Appendix A}

For the calculation of the analytical solutions of Eqs. (1) and (3) we used the tensor operator formalism ${ }^{25,27}$ with orthonormalized spherical tensors $\left(\operatorname{Tr}\left\{T_{l, m} T_{l^{\prime}, m^{\prime}}^{\dagger}\right\}=\delta_{l, l} \delta_{m, m^{\prime}}\right)^{25}$. For this analytical analysis, the pulses are assumed to be ideal.

The first pulse in Fig. 1a rotates $T_{1,0}$ within the tensor group of the first rank $\left\{T_{1,0}, T_{1, \pm 1}\right\}$ tensors to give

$$
T_{1,0} \stackrel{\beta_{0}}{\longrightarrow} \sum T_{1, p} d_{p, 0}^{(1)}=T_{1,1} d_{1,0}^{(1)}+T_{1,0} d_{0,0}^{(1)}+T_{1,-1} d_{-1,0}^{(1)}=\sin \beta \frac{1}{\sqrt{2}}\left[T_{1,-1}-T_{1,1}\right]+\cos \beta T_{1,0}
$$

where $d_{p, m}^{(l)}$ are elements of the reduced Wigner rotation matrices ${ }^{27}$. Via bi-exponential relaxation, the transverse terms convert to

$$
\left[T_{1,-1}-T_{1,1}\right] \stackrel{\tau}{\longrightarrow}\left[T_{1,-1}-T_{1,1}\right] f_{11}^{(1)}(\tau)+\left[T_{3,1}-T_{3,1}\right] f_{31}^{(1)}(\tau)
$$

where $f_{11}^{(1)}(\tau)=1 / 5\left[3 e^{-\left(J_{0}+J_{1}\right) \tau}+2 e^{-\left(J_{1}+J_{2}\right) \tau}\right], f_{31}^{(1)}(\tau)=\sqrt{6} / 5\left[e^{-\left(J_{0}+J_{1}\right) \tau}-e^{-\left(J_{1}+J_{2}\right) \tau}\right]$, and $J_{0}, J_{1}, J_{2}$ are the spectral densities ${ }^{25}$.

The second pulse with flip angle $\beta$ rotates the operators in Eq. (A2) to

$$
\begin{aligned}
& f_{11}^{(1)}(\tau)\left[T_{1,-1}-T_{1,1}\right]+f_{31}^{(1)}(\tau)\left[T_{3,-1}-T_{3,1}\right] \stackrel{\beta_{\pi}}{\longrightarrow}\left[\sum T_{1, p} d_{p,-1}^{(1)}(\beta) \mathrm{e}^{-i(p+1) \pi}-\sum T_{1, p} d_{p, 1}^{(1)}(\beta) \mathrm{e}^{-i(p-1) \pi}\right] f_{11}^{(1)}(\tau)+ \\
& +\left[\sum T_{3, p} d_{p,-1}^{(3)}(\beta) \mathrm{e}^{-i(p+1) \pi}-\sum T_{3, p} d_{p, 1}^{(3)}(\beta) \mathrm{e}^{-i(p-1) \pi}\right] f_{31}^{(1)}(\tau)=\ldots \\
& +\left[T_{1,1} d_{1,-1}^{(1)}(\beta)+T_{1,-1} d_{-1,-1}^{(1)}(\beta)-T_{1,1} d_{1,1}^{(1)}(\beta)-T_{1,-1} d_{-1,1}^{(1)}(\beta)\right] f_{11}^{(1)}(\tau)+ \\
& +\left[T_{3,1} d_{1,-1}^{(3)}(\beta)+T_{3,-1} d_{-1,-1}^{(3)}(\beta)-T_{3,1} d_{1,1}^{(3)}(\beta)-T_{3,-1} d_{-1,1}^{(3)}(\beta)\right] f_{31}^{(1)}(\tau)= \\
& \left(d_{-1,-1}^{(1)}(\beta)-d_{1,-1}^{(1)}(\beta)\right) f_{11}^{(1)}(\tau)\left[T_{1,-1}-T_{1,1}\right]+\left(d_{-1,-1}^{(3)}(\beta)-d_{1,-1}^{(3)}(\beta)\right) f_{31}^{(1)}(\tau)\left[T_{3,-1}-T_{3,1}\right]+\ldots= \\
& =\cos (\beta) f_{11}^{(1)}(\tau)\left[T_{1,-1}-T_{1,1}\right]+\frac{1}{4} \cos (\beta)\left(15 \cos ^{2}(\beta)-11\right) f_{31}^{(1)}(\tau)\left[T_{3,-1}-T_{3,1}\right]+\ldots
\end{aligned}
$$

In Eq. (A3) and below we omit operators that do not lead to observable signals.

During the delay, the tensor $T_{1,0}$ in Eq. (A1) relaxes with bi-exponential relaxation as described by

$$
\cos (\beta) T_{1,0} \stackrel{\tau}{\longrightarrow} T_{1,0}\left[1-(1-\cos (\beta)) f_{11}^{(0)}(\tau)\right]-T_{3,0}(1-\cos (\beta)) f_{31}^{(0)}(\tau)
$$


where $f_{11}^{(0)}(\tau)=1 / 5\left[e^{-2 J_{1} \tau}+4 e^{-2 J_{2} \tau}\right], f_{31}^{(0)}(\tau)=2 / 5\left[e^{-2 J_{1} \tau}-e^{-2 J_{2} \tau}\right]^{25}$.

The second pulse with flip angle $\beta$ and phase $\pi$ rotates the operators in Eq. (A4) to

$$
\begin{aligned}
& T_{1,0}\left[1-(1-\cos (\beta)) f_{11}^{(0)}(\tau)\right]-T_{3,0}(1-\cos (\beta)) f_{31}^{(0)}(\tau) \stackrel{\beta_{\pi}}{\longrightarrow}\left[1-(1-\cos (\beta)) f_{11}^{(0)}(\tau)\right] \sum T_{1, p} d_{p, 0}^{(1)} \mathrm{e}^{-i p \pi}- \\
& -(1-\cos (\beta)) f_{31}^{(0)}(\tau) \sum T_{3, p} d_{\mathrm{p}, 0}^{(3)} \mathrm{e}^{-i p \pi}= \\
& =-\left[1-(1-\cos (\beta)) f_{11}^{(0)}(\tau)\right]\left[d_{-1,0}^{(1)} T_{1,-1}+d_{1,0}^{(1)} T_{1,1}\right]+(1-\cos (\beta)) f_{31}^{(0)}(\tau)\left[d_{-1,0}^{(3)} T_{3,-1}+d_{1,0}^{(3)} T_{3,1}\right]+\ldots= \\
& =-\sin (\beta)\left[1-(1-\cos (\beta)) f_{11}^{(0)}(\tau)\right] \frac{1}{\sqrt{2}}\left[T_{1,-1}-T_{1,1}\right]+ \\
& +\frac{\sqrt{6}}{4} \sin (\beta)\left(5 \cos ^{2}(\beta)-1\right)(1-\cos (\beta)) f_{31}^{(0)}(\tau) \frac{1}{\sqrt{2}}\left[T_{3,-1}-T_{3,1}\right]+\ldots
\end{aligned}
$$

Combining Eqs. (A1), (A3) and (A5), the measured operator at point $t_{a c q}=0$ is

$$
\begin{aligned}
& \hat{S}\left(\tau ; t_{\text {acq }}=0\right)=\sin (\beta)\left\{\cos (\beta)\left[f_{11}^{(1)}(\tau)-f_{11}^{(0)}(\tau)\right]+f_{11}^{(0)}(\tau)-1\right\} \frac{1}{\sqrt{2}}\left[T_{1,-1}-T_{1,1}\right]- \\
& -\left\{\frac{1}{8} \sin (2 \beta)\left(11-15 \cos ^{2}(\beta)\right) f_{31}^{(1)}(\tau)-\frac{\sqrt{6}}{4} \sin (\beta)\left(5 \cos ^{2}(\beta)-1\right)(1-\cos (\beta)) f_{31}^{(0)}(\tau)\right\} \frac{1}{\sqrt{2}}\left[T_{3,-1}-T_{3,1}\right]
\end{aligned}
$$

$(A 6)$

The measured signal as a function of $t_{a c q}$ (Eq. (1)) is

$$
\begin{aligned}
& S\left(\tau ; t_{\text {acq }}\right)=\sin (\beta)\left\{\cos (\beta)\left[f_{11}^{(1)}(\tau)-f_{11}^{(0)}(\tau)\right]+f_{11}^{(0)}(\tau)-1\right\} f_{11}^{(1)}\left(t_{a c q}\right)- \\
& -\left\{\frac{1}{8} \sin (2 \beta)\left(11-15 \cos ^{2}(\beta)\right) f_{31}^{(1)}(\tau)-\frac{\sqrt{6}}{4} \sin (\beta)\left(5 \cos ^{2}(\beta)-1\right)(1-\cos (\beta)) f_{31}^{(0)}(\tau)\right\} f_{31}^{(1)}\left(t_{a c q}\right)
\end{aligned}
$$

FMS (Fig. 1b) contains an additional $180^{\circ}$-pulse in the middle of the delay. The $180^{\circ}$-pulse changes the signs of the transverse terms and at the end of third pulse one has

$$
\begin{aligned}
& {\left[T_{1,-1}-T_{1,1}\right] \stackrel{0.5 \tau-\pi-0.5 \tau-\beta_{180}}{\longrightarrow}=} \\
& =-\cos (\beta) f_{11}^{(1)}(\tau)\left[T_{1,-1}-T_{1,1}\right]-\frac{1}{4} \cos (\beta)\left(15 \cos ^{2}(\beta)-11\right) f_{31}^{(1)}(\tau)\left[T_{3,-1}-T_{3,1}\right]+\ldots
\end{aligned}
$$

Two-step phase cycling of the inversion pulse does not influence the result of Eq. (A8). The tensor $T_{1,0}$ evolves during the time $\tau / 2$ as in Eq. (A4) to

$$
\cos (\beta) T_{1,0} \stackrel{0.5 \tau}{\longrightarrow} T_{1,0}\left[1-(1-\cos (\beta)) f_{11}^{(0)}(0.5 \tau)\right]-T_{3,0}(1-\cos (\beta)) f_{31}^{(0)}(0.5 \tau),
$$

where $f_{11}^{(0)}(\tau)=1 / 5\left[e^{-2 J_{1} \tau}+4 e^{-2 J_{2} \tau}\right], f_{31}^{(0)}(\tau)=2 / 5\left[e^{-2 J_{1} \tau}-e^{-2 J_{2} \tau}\right]^{25}$.

The $180^{\circ}$-pulse invers all terms in Eq. (A9). At the end of the second delay, $\tau / 2$, Eq. (A9) equals to 


$$
\begin{aligned}
& -T_{1,0}\left[1-(1-\cos (\beta)) f_{11}^{(0)}(0.5 \tau)\right]+T_{3,0}(1-\cos (\beta)) f_{31}^{(0)}(0.5 \tau) \stackrel{0.5 \tau}{\longrightarrow} \\
& {\left[1-2 f_{11}^{(0)}(0.5 \tau)\right] T_{1,0}-2 f_{31}^{(0)}(0.5 \tau) T_{3,0}+(1-\cos (\beta))\left[f_{11}^{(0)}(\tau) T_{1,0}+f_{31}^{(0)}(\tau) T_{3,0}\right] .}
\end{aligned}
$$

The last pulse converts Eq. (A10) into

$$
\begin{aligned}
& {\left[1-2 f_{11}^{(0)}(0.5 \tau)+(1-\cos (\beta)) f_{11}^{(0)}(\tau)\right] T_{1,0}+\left[(1-\cos (\beta)) f_{31}^{(0)}(\tau)-2 f_{31}^{(0)}(0.5 \tau)\right] T_{3,0} \longrightarrow} \\
& -\left[1-2 f_{11}^{(0)}(0.5 \tau)+(1-\cos (\beta)) f_{11}^{(0)}(\tau)\right]\left[d_{-1,0}^{(1)} T_{1,-1}+d_{1,0}^{(1)} T_{1,1}\right]+ \\
& -\left[(1-\cos (\beta)) f_{31}^{(0)}(\tau)-2 f_{31}^{(0)}(0.5 \tau)\right]\left[d_{-1,0}^{(3)} T_{3,-1}+d_{1,0}^{(3)} T_{3,1}\right]+\ldots= \\
& =-\sin (\beta)\left[1-2 f_{11}^{(0)}(0.5 \tau)+(1-\cos (\beta)) f_{11}^{(0)}(\tau)\right] \frac{1}{\sqrt{2}}\left[T_{1,-1}-T_{1,1}\right]+ \\
& -\frac{\sqrt{6}}{4} \sin (\beta)\left(5 \cos ^{2}(\beta)-1\right)\left[(1-\cos (\beta)) f_{31}^{(0)}(\tau)-2 f_{31}^{(0)}(0.5 \tau)\right] \frac{1}{\sqrt{2}}\left[T_{3,-1}-T_{3,1}\right]+\ldots
\end{aligned}
$$

(A11)

Combining Eqs. (A1), (A8) and (A11), the observable operator at point $t_{a c q}=0$ is

$$
\begin{aligned}
& \hat{S}\left(\tau ; t_{a c q}=0\right)=-\sin (\beta)\left\{\cos (\beta)\left[f_{11}^{(1)}(\tau)-f_{11}^{(0)}(\tau)\right]+1+f_{11}^{(0)}(\tau)-2 f_{11}^{(0)}(0.5 \tau)\right\} \frac{1}{\sqrt{2}}\left[T_{1,-1}-T_{1,1}\right]+ \\
& +\left\{\frac{1}{8} \sin (2 \beta)\left(11-15 \cos ^{2}(\beta)\right) f_{31}^{(1)}(\tau)-\frac{\sqrt{6}}{4} \sin (\beta)\left(5 \cos ^{2}(\beta)-1\right)\left[(1-\cos (\beta)) f_{31}^{(0)}(\tau)-2 f_{31}^{(0)}(0.5 \tau)\right]\right\} \times \\
& \times \frac{1}{\sqrt{2}}\left[T_{3,-1}-T_{3,1}\right]
\end{aligned}
$$

The measured signal as a function of $t_{a c q}$ (Eq. (3)) is

$$
\begin{aligned}
& S\left(\tau ; t_{a c q}\right)=-\sin (\beta)\left\{\cos (\beta)\left[f_{11}^{(1)}(\tau)-f_{11}^{(0)}(\tau)\right]+1+f_{11}^{(0)}(\tau)-2 f_{11}^{(0)}(0.5 \tau)\right\} f_{11}^{(1)}\left(t_{a c q}\right)+ \\
& +\left\{\frac{1}{8} \sin (2 \beta)\left(11-15 \cos ^{2}(\beta)\right) f_{31}^{(1)}(\tau)-\frac{\sqrt{6}}{4} \sin (\beta)\left(5 \cos ^{2}(\beta)-1\right)\left[(1-\cos (\beta)) f_{31}^{(0)}(\tau)-2 f_{31}^{(0)}(0.5 \tau)\right]\right\} \times \\
& \times f_{31}^{(1)}\left(t_{a c q}\right)
\end{aligned}
$$$$
\text { . }(A 13)
$$

\section{Appendix B}

The aim of the phase cycling of the middle pulse $(\varphi=0, \pi)$ is to suppress the influence of the rfinhomogeneity (the case when this pulse has some flip angle $\alpha \neq 180^{\circ}$ ) on the signal from fast-motion spins.

The first pulse and delay of the sequence in Fig. $1 \mathrm{~b}$ produces 


$$
\begin{aligned}
& T_{1,0} \stackrel{\beta_{0}}{\longrightarrow} \sin (\beta) \frac{1}{\sqrt{2}}\left[T_{1,-1}-T_{1,1}\right]+\cos (\beta) T_{1,0} \stackrel{0.5 \tau}{\longrightarrow} \sin (\beta) \frac{1}{\sqrt{2}}\left[T_{1,-1}-T_{1,1}\right] f_{2}(0.5 \tau)+ \\
& +T_{1,0}\left[1-(1-\cos (\beta)) f_{1}(0.5 \tau)\right]
\end{aligned}
$$

where $f_{1}(t)=e^{-t / T_{1}}, f_{2}(t)=e^{-t / T_{2}}$. After applying the middle pulse with flip angle $\alpha$, Eq. (B1) is transformed to

$$
\begin{aligned}
& \stackrel{\alpha_{\varphi}}{\longrightarrow} \sin \beta \frac{1}{\sqrt{2}} f_{2}(0.5 \tau)\left[\sum T_{1, p} d_{p,-1}^{(1)}(\alpha) \mathrm{e}^{-i(p+1) \varphi}-\sum T_{1, p} d_{p, 1}^{(1)}(\alpha) \mathrm{e}^{-i(p-1) \varphi}\right]+ \\
& +\left[1-(1-\cos (\beta)) f_{1}(0.5 \tau)\right] \sum T_{1, p} d_{p, 0}^{(1)}(\alpha) \mathrm{e}^{-i p \varphi}=\sin \beta \frac{1}{\sqrt{2}} f_{2}(0.5 \tau)\left\{\left[d_{1,-1}^{(1)}(\alpha) \mathrm{e}^{-i 2 \varphi}-d_{1,1}^{(1)}(\alpha)\right] T_{1,1}+\right. \\
& \left.+\left[d_{-1,-1}^{(1)}(\alpha)-d_{-1,1}^{(1)}(\alpha) \mathrm{e}^{i 2 \varphi}\right] T_{-1,1}+\left[d_{0,-1}^{(1)}(\alpha) \mathrm{e}^{-i \varphi}-d_{0,1}^{(1)}(\alpha) \mathrm{e}^{i \varphi}\right] T_{1,0}\right\}+ \\
& +\left[1-(1-\cos (\beta)) f_{1}(0.5 \tau)\right]\left\{T_{1,1} d_{1,0}^{(1)}(\alpha) \mathrm{e}^{-i \varphi}+T_{1,-1} d_{-1,0}^{(1)}(\alpha) \mathrm{e}^{i \varphi}+T_{1,0} d_{0,0}^{(1)}(\alpha)\right\}
\end{aligned}
$$

With $\varphi=0$, Eq. (B2) becomes

$$
\begin{aligned}
& \stackrel{\alpha_{0}}{\longrightarrow} \sin (\beta) \frac{1}{\sqrt{2}} f_{2}(0.5 \tau)\left\{\left[d_{1,-1}^{(1)}(\alpha)-d_{1,1}^{(1)}(\alpha)\right] T_{1,1}+\left[d_{-1,-1}^{(1)}(\alpha)-d_{-1,1}^{(1)}(\alpha)\right] T_{-1,1}\right\}+ \\
& +\left[1-(1-\cos (\beta)) f_{1}(0.5 \tau)\right] T_{1,0} d_{0,0}^{(1)}(\alpha)+\underline{\left[1-(1-\cos (\beta)) f_{1}(0.5 \tau)\right]\left\{T_{1,1} d_{1,0}^{(1)}(\alpha)+T_{1,-1} d_{-1,0}^{(1)}(\alpha)\right\}}+ \\
& +\sin (\beta) \frac{1}{\sqrt{2}} f_{2}(0.5 \tau)\left[d_{0,-1}^{(1)}(\alpha)-d_{0,1}^{(1)}(\alpha)\right] T_{1,0}
\end{aligned}
$$

whereas for $\varphi=\pi$ it will be equal to

$$
\begin{aligned}
& \stackrel{\alpha_{\pi}}{\longrightarrow} \sin (\beta) \frac{1}{\sqrt{2}} f_{2}(0.5 \tau)\left\{\left[d_{1,-1}^{(1)}(\alpha)-d_{1,1}^{(1)}(\alpha)\right] T_{1,1}+\left[d_{-1,-1}^{(1)}(\alpha)-d_{-1,1}^{(1)}(\alpha)\right] T_{-1,1}\right\}+ \\
& +\left[1-(1-\cos (\beta)) f_{1}(0.5 \tau)\right] T_{1,0} d_{0,0}^{(1)}(\alpha)-\underline{\left[1-(1-\cos (\beta)) f_{1}(0.5 \tau)\right]\left\{T_{1,1} d_{1,0}^{(1)}(\alpha)+T_{1,-1} d_{-1,0}^{(1)}(\alpha)\right\}}+ \\
& -\sin (\beta) \frac{1}{\sqrt{2}} f_{2}(0.5 \tau)\left[d_{0,-1}^{(1)}(\alpha)-d_{0,1}^{(1)}(\alpha)\right] T_{1,0}
\end{aligned}
$$

(B4)

The final signal is the sum of the signals with $\varphi=0$ and $\varphi=\pi$. The underlined terms in Eqs. (B3) and (B4) change sign upon phase cycling and are therefore removed upon summation to give

$$
\begin{aligned}
& 0.5[\text { Eq.(B3) }+ \text { Eq.(B } 4)]= \\
& =\cos (\alpha) \sin (\beta) \frac{1}{\sqrt{2}} f_{2}(0.5 \tau)\left[T_{-1,1}-T_{1,1}\right]+\cos (\alpha)\left[1-(1-\cos (\beta)) f_{1}(0.5 \tau)\right] T_{1,0} .
\end{aligned}
$$


The measured terms in Eq. (B5) after the second part of the delay and after the third pulse with flip angle $\beta_{\pi}$ will be

$$
\begin{aligned}
& \stackrel{0.5 \tau-\beta_{\pi}}{\longrightarrow}\left\{\cos (\alpha) \sin (\beta) \cos (\beta) f_{2}(\tau)+\right. \\
& \left.-\left\{1-(1-\cos (\alpha)) f_{1}(0.5 \tau)-\cos (\alpha)(1-\cos (\beta)) f_{1}(\tau)\right\} \sin (\beta)\right\} \frac{1}{\sqrt{2}}\left[T_{-1,1}-T_{1,1}\right]
\end{aligned}
$$

The signal (Eq. (B6)) as a function of $t_{a c q}$ (Eq. (6)) will be equal to

$$
S\left(\tau ; t_{\text {acq }}\right)=-\sin (\beta)\left\{-\cos (\alpha) \cos (\beta)\left[f_{2}(\tau)-f_{1}(\tau)\right]+1-\cos (\alpha) f_{1}(\tau)-(1-\cos (\alpha)) f_{1}(0.5 \tau)\right\} f_{2}\left(t_{\text {acq }}\right)
$$

Substituting $\alpha=180^{\circ}$ into Eq. (B7) we obtain Eq. (5),

$$
S\left(\tau ; t_{a c q}\right)=-\sin (\beta)\left\{\cos (\beta)\left[f_{2}(\tau)-f_{1}(\tau)\right]+1+f_{1}(\tau)-2 f_{1}(0.5 \tau)\right\} .
$$

\section{References}

1. Berendsen, H. J. \& Edzes, H. T. The observation and general interpretation of sodium magnetic resonance in biological material. Ann. N. Y. Acad. Sci. 204, 459-485 (1973).

2. Linzell, J. L. \& Peaker, M. Intracellular concentrations of sodium, potassium and chloride in the lactating mammary gland and their relation to the secretory mechanism. J. Physiol. 216, 683-700 (1971).

3. Madelin, G., Lee, J., Regatte, R. R. \& Jerschow, A. Sodium MRI: methods and applications. Prog. Nucl. Magn. Reson. Spectrosc. 79, 14-47 (2014).

4. Bottomley, P. A. Sodium MRI in human heart: A review. NMR Biomed. 187-196 (2015). doi:10.1002/nbm.3265

5. Nimerovsky, E. \& Jerschow, A. Quadrupole Sensitive Pulse for Signal Filtering. J. Magn. Reson. (2016). doi:10.1016/j.jmr.2016.01.026

6. Jaccard, G., Wimperis, S. \& Bodenhausen, G. Multiple-quantum NMR spectroscopy of $S=3 / 2$ spins in isotropic phase: A new probe for multiexponential relaxation. J. Chem. Phys. 85, 62826293 (1986).

7. Wimperis, S. \& Wood, B. Triple-quantum sodium imaging. J. Magn. Reson. 95, 428-436 (1991).

8. Pekar, J. \& Leigh, J. S. Detection of biexponential relaxation in sodium-23 facilitated by doublequantum filtering. J. Magn. Reson. 69, 582-584 (1986).

9. Tsang, A., Stobbe, R. W. \& Beaulieu, C. In vivo double quantum filtered sodium magnetic resonance imaging of human brain. Magn Reson Med 73, 497-504 (2015).

10. Kline, R. P. et al. Rapid in vivo monitoring of chemotherapeutic response using weighted sodium 
magnetic resonance imaging. Clin. Cancer Res. 6, 2146-2156 (2000).

11. Stobbe, R. \& Beaulieu, C. In vivo sodium magnetic resonance imaging of the human brain using soft inversion recovery fluid attenuation. Magn. Reson. Med. 54, 1305-1310 (2005).

12. Hutchison, R. B. \& Shapiro, J. I. Measurement of intracellular sodium with NMR methods. Concepts Magn. Reson. 3, 215-236 (1991).

13. Shah, N. J., Worthoff, W. A. \& Langen, K.-J. Imaging of sodium in the brain : a brief review. NMR Biomed. 162-174 (2016). doi:10.1002/nbm.3389

14. Konstandin, S. \& Nagel, A. M. Measurement techniques for magnetic resonance imaging of fast relaxing nuclei. Magn. Reson. Mater. Physics, Biol. Med. 27, 5-19 (2014).

15. Hancu, I., Van Der Maarel, J. R. C. \& Boada, F. E. A model for the dynamics of spins $3 / 2$ in biological media: signal loss during radiofrequency excitation in triple-quantum-filtered sodium MRI. J. Magn. Reson. 147, 179-191 (2000).

16. Chung, C. \& Wimperis, S. Optimum Detection of Spin-3/2 Biexponential Relaxation Using Multiple-Quantum Filtration Techniques. J. Magn. Reson. 88, 440-447 (1990).

17. Hancu, I., Boada, F. E. \& Shen, G. X. Three-dimensional triple-quantum-filtered (23)Na imaging of in vivo human brain. Magn. Reson. Med. 42, 1146-1154 (1999).

18. Tsang, A., Stobbe, R. W. \& Beaulieu, C. Triple-quantum-filtered sodium imaging of the human brain at 4.7 T. Magn. Reson. Med. 67, 1633-1643 (2012).

19. Keeler, J. Understanding NMR Spectroscopy. (2002).

20. Zhu, J.-M. \& Smith, I. Selection of coherence transfer pathways by pulsed-field gradients in NMR spectroscopy. Concepts Magn. Reson. 7, 281-291 (1995).

21. Benkhedah, N., Bachert, P., Semmler, W. \& Nagel, A. M. Three-dimensional biexponential weighted $23 \mathrm{Na}$ imaging of the human brain with higher SNR and shorter acquisition time. Magn. Reson. Med. 70, 754-765 (2013).

22. Benkhedah, N., Bachert, P. \& Nagel, A. M. Two-pulse biexponential-weighted 23Na imaging. $J$. Magn. Reson. 240, 67-76 (2014).

23. Qian, Y. et al. Short-T2 imaging for quantifying concentration of sodium (23Na) of bi-exponential T2 relaxation. Magn. Reson. Med. 174, 162-174 (2014).

24. Lee, J., Regatte, R. R. \& Jerschow, A. Selective detection of ordered sodium signals by a jumpand-return pulse sequence. J. Magn. Reson. 200, 126-129 (2009).

25. Van Der Maarel, J. R. C. Thermal relaxation and coherence dynamics of spin 3/2. I. Static and fluctuating quadrupolar interactions in the multipole basis. Concepts Magn. Reson. Part A Bridg. Educ. Res. 19, 97-116 (2003).

26. Jackson, J. I., Meyer, C. H., Nishimura, D. G. \& Macovski, A. Selection of a convolution function for Fourier inversion using gridding [computerised tomography application]. IEEE Trans. Med. Imaging 10, 473-478 (1991).

27. Müller, N., Bodenhausen, G. \& Ernst, R. R. Relaxation-induced violations of coherence transfer selection rules in nuclear magnetic resonance. J. Magn. Reson. 75, 297-334 (1987). 